\title{
ON THE MEASUREMENT AND PREDICTION OF RAINFALL NOISE
}

\author{
G. Schmid ${ }^{1}$, M. J. Kingan ${ }^{1}$ L. Panton ${ }^{2}$, G. Willmott ${ }^{2,3,4}$, Y. Yang ${ }^{1}$, C. Decraene ${ }^{5}$, E. Reynders ${ }^{5}$, A. \\ Hall $^{1}$ \\ ${ }^{1}$ Acoustics Research Centre, Department of Mechanical Engineering, University of Auckland, \\ Auckland 1010, New Zealand. \\ ${ }^{2}$ Department of Physics, University of Auckland, Auckland 1010, New Zealand. \\ ${ }^{3}$ School of Chemical Sciences, University of Auckland, Auckland 1010, New Zealand. \\ ${ }^{4}$ The MacDiarmid Institute for Advanced Materials and Nanotechnology \\ ${ }^{5}$ University of Leuven (KU Leuven), Department of Civil Engineering, Kasteelpark Arenberg 40, 3001 \\ Leuven, Belgium
}

\begin{abstract}
This paper concerns the measurement and prediction of rainfall noise with a particular focus on measurements made using the method described in the ISO rainfall noise standard. Rainfall noise is generated by rain impacting on a surface which excites the surface producing noise. Here, several different models for the force produced by a water droplet impacting on a flat inclined surface are presented. These models are designed for the nominal intense and heavy raindrops described in the ISO standard and are validated against experimental measurements. The best-performed models have been incorporated into theoretical methods for predicting the noise produced by rainfall on a flat inclined panel. These methods are used to produce noise level predictions for heavy rainfall on a standard reference test specimen which are compared with experimental measurements and show moderate agreement. These experiments were conducted using the test method described in the ISO standard. Several issues which we encountered implementing this test method are described and a number of suggestions are made to improve this method. Finally, the theoretical models are used to investigate the effect of different parameters on rainfall noise for the purpose of illustrating potential sources of error during testing using the ISO standard method.
\end{abstract}

\section{Introduction}

The forces produced when raindrops impact on a roof can be a significant source of noise, particularly for lightweight rooves [1]. The forces produced by the raindrop impacts induce vibration in the roof structure producing sound, which can be very disruptive to the occupants below if there is insufficient insulation. For this reason, standards for some buildings (e.g. school buildings in New Zealand and the UK [2], [3]) attempt to ensure that roof systems are constructed to produce rain noise of an acceptably low level. The rain noise produced by a roof system (or a component of a roof system - e.g. a skylight) can be assessed experimentally using the methods described in ISO 10140-5:2010/Amd.1:2014 [4] and Annex K of ISO 10140-1:2016 [5].

Rain noise has also been the subject of a number of academic investigations over the years. In 1969, Dubout [6] experimentally investigated the effect of rainfall rate on the sound pressure level produced in a room beneath an unlined, low-slope, steel-trough roof and derived an empirical relationship 
between the sound intensity level radiated from the roof and the rainfall rate. Ballagh [1] presented an experimental method for measuring the noise produced by 'simulated rainfall' on a roof. That work also proposed a theoretical model for predicting rain noise. McLoughlin, Saunders and Ford [7] then described an experimental method for determining the sound intensity radiated from roof structures excited by artificial rainfall in a laboratory. They used this method to measure the sound intensity produced by rainfall on a number of different rooves and to deduce simple empirical formulae relating the sound intensity to the droplet size, impact velocity and rainfall rate for rainfall on a 'single-skin profiled roof'. Suga and Tachibana [8] conducted an experimental investigation of the sound power produced by artificial rainfall on different lightweight roof systems. They also observed that there was an approximately linear relationship between the sound power and the sound transmission loss for the rooves they investigated. Vermeir, Mees and Bosmans [9] present the results of an experimental and theoretical study investigating the sound power radiated from different glass panels subjected to artificial rainfall. Guigou-Carter, Villot and Horlaville [10] and Hopkins [11] have presented similar theoretical methods for calculating rain noise. More recently, Griffin and Ballagh [12] have presented an engineering model for rain noise prediction. Toyoda and Takahashi [13] presented an analytical model for predicting the noise produced by rainfall on an 'Ethylene/TetraFluoro Ethylene (ETFE) skin roof' and compared their predictions with experiments conducted using a method based on the ISO rainfall noise standard (refs. [4] and [5]). Yan, $\mathrm{Lu}$ and $\mathrm{Li}$ [14] conducted a detailed experimental investigation into the noise produced by both natural and artificial rain on a roof and noted that the wide range of natural rainfall rates and droplet sizes can result in significant variations in noise which are quite different to those simulated using the artificial rain specified in the ISO rainfall noise standard. They also presented empirical formulae for predicting rain noise due to natural rainfall.

The accurate prediction of rainfall noise requires several phenomena to be accurately modelled. Firstly, the rainfall must be characterised in terms of its rainfall rate, raindrop size and impact velocity. When following the ISO rainfall noise standards [4],[5], these parameters are specified for the artificial rainfall used and therefore modelling of these characteristics is of secondary importance.

Secondly, the forces produced by the raindrop impacts must be accurately modelled. The rain noise prediction methods described in refs. [1], [10]-[12] incorporate simple droplet impact force models based on that of Petersson [15], [16]. Recently, a number of empirical and semi-empirical models for predicting impact forces have been developed using accurate measurements of forces produced by droplets impacting on flat and inclined surfaces (see refs. [17]-[21]). In particular, Yu and Hopkins [21] used several such advanced models to predict the structure-borne sound power input to a surface from artificial and natural rainfall. Their models account for the inclination of the impact surface and the possibility of a thin layer of water on the impact surface. In addition to these, we note that other studies have experimentally investigated the force produced by droplets impacting on a surface. Examples include Nearing and Bradford [22], Nearing, Bradford and Holtz [23], Grinspan \& Gnanamoorthy [24], Li et al. [25], Soto et al. [26] and Zhang et al. [27].

Finally, rainfall noise prediction requires the accurate prediction of the response of and acoustic radiation from the impact surface. Refs. [10] and [11] presented a statistical energy analysis (SEA) method based on that described by Craik [28] to predict the radiated sound power level. Guigou-Carter, Villot and Horlaville [10] also described several alternative methods for calculating the sound power radiated from the roof structure including a multi-layered structure model combined with a spatial windowing technique [29] and a modal method based on the model described in [30]. Ref. [12] presented a method for calculating the radiated sound power which includes both the resonant and nonresonant vibration of the surface. 
The main aims of this work are to measure drop impact forces and rainfall noise in laboratory experiments based on the ISO rainfall noise standard, to develop state-of-the-art theoretical models for predicting this noise, and to use these models to investigate the effect of different parameters on rainfall noise. An auxiliary goal of the paper is to investigate issues with implementation of the ISO standard method, and to discuss ways in which it could be improved. In $\S \S 2.1-2$ the types of rain used in the test method are discussed and several models for predicting the impact force produced by this rainfall onto a flat inclined surface are described. In $\$ 2.3$ the accuracy of these models is assessed by comparing predictions made using them with the results of a detailed experimental study. In $\S 3$ these impact force models are incorporated into theoretical models for predicting the noise produced by rainfall on a flat inclined panel. In $\S 4$ an experimental study is described in which the noise produced by heavy rainfall on the standard reference test specimen described in the ISO standard was measured using the method also described in the standard. In $\S 4.1$ we describe some of the issues we faced implementing this test method and make several suggestions for future work to further investigate and improve this method. The measured levels are also compared with theoretical predictions and show moderate agreement. In $\S 4.2$, the theoretical models are used to investigate the effect of different parameters on rainfall noise for this purpose of illustrating potential sources of error during testing using the ISO standard method.

\section{Droplet impact forces}

\subsection{Droplet size, shape and impact velocity}

The ISO rainfall noise standard uses a water tank with a perforated base to generate artificial rain. The standard specifies that either 'heavy' or 'intense' rain should be used during testing and that the rainfall rate, droplet diameter and impact velocity are $15 \mathrm{~mm} \cdot \mathrm{h}^{-1}, 2 \mathrm{~mm}$ and $4 \mathrm{~m} \cdot \mathrm{s}^{-1}$ respectively for intense rain and $40 \mathrm{~mm} \cdot \mathrm{h}^{-1}, 5 \mathrm{~mm}$ and $7 \mathrm{~m} \cdot \mathrm{s}^{-1}$ for heavy rain ${ }^{1}$. Note that 'impact velocity' is defined as the velocity of the droplet at the instant just before impact. Testing using heavy rain is mandatory whilst intense rain is only recommended if "lower rainfall rates are needed". The standard requires that $50 \%$ of droplets have diameters within $\pm 0.5 \mathrm{~mm}$ and impact velocities within $\pm 1 \mathrm{~m} \cdot \mathrm{s}^{-1}$ of these nominal values. The ISO standard does not define how "droplet diameter" is to be interpreted (despite the fact that heavy rain will not have a spherical shape on impact). Following Chene, Guigou-Carter and Larsen [31] and Yu and Hopkins [21], we interpret droplet diameter as the diameter of a sphere of equal volume to that of the droplet.

In this paper we assume, unless otherwise stated, that water droplets are at $20^{\circ} \mathrm{C}$ and $101.325 \mathrm{kPa}$ in air at the same state. For such a case, ref. [32] gives $\rho_{w}=998$. kg.m ${ }^{-3}, \mu_{w}=1.003 \times 10^{-3}$ Pa.s, $\rho_{a}=$ $1.20 \mathrm{~kg} . \mathrm{m}^{-3}$. The speed of sound is taken to be $c_{0}=343 \mathrm{~m} \cdot \mathrm{s}^{-1}$. These values are used throughout this paper.

$\mathrm{Yu}$ and Hopkins [19] measured the impact velocity for $2 \mathrm{~mm}$ diameter and $4.5 \mathrm{~mm}$ diameter water droplets falling in air from various heights between $0.41 \mathrm{~m}$ and $15 \mathrm{~m}$. They compared their measurements with those predicted using the empirical model of Range and Feuillebois [33] which

\footnotetext{
${ }^{1}$ Note that table $\mathrm{K} 1$ in Annex K of ref. [5] gives four rainfall type classifications (moderate, intense, heavy and cloudburst). These are attributed to IEC 60721-2-2. However, these classification names and the rainfall characteristics (rainfall rate, typical drop diameter and fall velocity) do not correspond with those given in the current version of IEC 60721-2-2 (published in 2012) which lists 'heavy rain' as having a rainfall rate of $<16$ $\mathrm{mm} \cdot \mathrm{h}^{-1}$ and 'very heavy rain' as having a rainfall rate $<50 \mathrm{~mm} \cdot \mathrm{h}^{-1}$. No characteristics, other than rain intensity are associated with the different rain classifications in IEC 60721-2-2. However, it is stated that rain, in general, has a droplet size distribution typically $1 \mathrm{~mm}$ to $2 \mathrm{~mm}$ in diameter, with diameters up to $5 \mathrm{~mm}$ to $8 \mathrm{~mm}$ in thunderstorms; fall velocities are stated as being typically between $2 \mathrm{~m} \cdot \mathrm{s}^{-1}$ and $12 \mathrm{~m} . \mathrm{s}^{-1}$.
} 
gives the impact velocity $U_{0}$, for a droplet with diameter $D$, density $\rho_{w}$ and viscosity $\mu_{w}$ falling through air with density $\rho_{a}$ from a height $H$ as

$$
U_{0}=\sqrt{\frac{g}{A}(1-\exp \{-2 A H\})}
$$

where $g=9.81 \mathrm{~m} \cdot \mathrm{s}^{-2}$ is the force per unit mass due to gravity and

$$
A=\frac{3 c_{f} \rho_{a}}{4 \rho_{w} D} .
$$

The friction coefficient $c_{f}$ in eq. (2) is dependent on Reynolds number

$$
\operatorname{Re}=\frac{\rho_{w} U_{0} D}{\mu_{w}}
$$

$\mathrm{Yu}$ and Hopkins found that using $c_{f}=0.533$ provided a good fit to their experimental data which covered a Reynolds number range of $5140<\operatorname{Re}<41265$ (which includes the nominal intense and heavy raindrops). Using $c_{f}=0.533$ in eq. (3) and rearranging for $H$ yields a drop height of $1.04 \mathrm{~m}$ to produce an impact velocity of $4 \mathrm{~m} \cdot \mathrm{s}^{-1}$ for the nominal $2 \mathrm{~mm}$ diameter intense rain droplet. This compares well with the recommended fall height of "approx. $1 \mathrm{~m}$ " stated in Table H.2 of the rainfall noise standard [4]. For a nominal $5 \mathrm{~mm}$ diameter heavy rain droplet, an impact velocity of $7 \mathrm{~m} \cdot \mathrm{s}^{-1}$ is predicted for a drop height of $3.41 \mathrm{~m}$. This also compares well with the recommended fall height of "approx. $3.5 \mathrm{~m}$ " stated in Table H.2 of the rainfall noise standard. Note that these impact velocities are somewhat less than terminal velocity (the maximum velocity at which the droplet would attain during freefall).

A number of different models exist for describing the shape of a water droplet falling in air. For example, Clift, Grace and Weber [34] propose an ellipsoidal shape model for a liquid drop moving through a gas at terminal velocity. The shape of water droplets falling through air with nominal diameters between $1 \mathrm{~mm}$ and $5 \mathrm{~mm}$ predicted using this model are shown in figure 1. Small rain drops are approximately spherical but larger drops are aspherical with flatter bottoms.

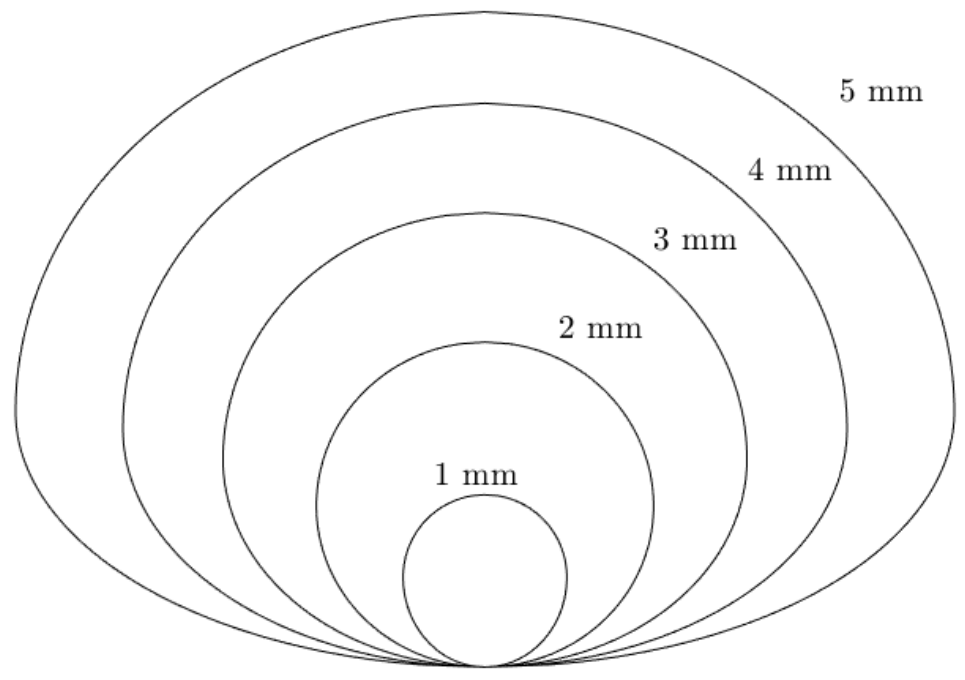

Figure 1. The shape of water droplets falling in air at terminal velocity for different nominal diameters (shown in text adjacent to the droplet). Calculated using the ellipsoidal model of Clift, Grace and Weber [34]. 


\subsection{Droplet impact force modelling}

The force exerted by a single droplet impact on a flat surface is denoted $F_{1}$. This force varies with time, $t$, and typically increases impulsively from the time of impact, at $t=0$, rising to a maximum a short time later and then decreasing relatively slowly to zero. Following Gordillo, Sung and Cheng [17] and $\mathrm{Yu}$ and Hopkins [21], we introduce a dimensionless force, $\widetilde{F}_{1}$, and time, $\tilde{t}$, which are related to their dimensional equivalents via

$$
F_{1}(t)=\rho_{w} D^{2} U_{\perp}^{2} \tilde{F}_{1}(\tilde{t}), \quad t=\frac{\tilde{t} D}{U_{\perp}}
$$

where $U_{\perp}=U_{0} \cos \theta$ is the velocity perpendicular to the flat impact surface which is inclined at angle $\theta$ to the horizontal.

Petersson [15], [16] describes a simple model for the force produced by a droplet impact on a horizontal plane surface. The model assumes that the droplet falls vertically downwards at constant velocity and that a particle within the droplet has identical vertical velocity until the time at which the particle would have passed through the plane. At this instant, the particle velocity is instantaneously redirected in a radial direction in the horizontal plane. This model predicts that droplet shapes with flatter bottoms will produce more impulsive forces as there is a higher rate of vertical-to-horizontal momentum transfer just after impact compared to later times. Although this model is a gross oversimplification, when different droplet shapes are used it yields impact forces which are quantitatively similar to measurements (see, for example, ref. [16]). This model has therefore been used in the rain noise models of Ballagh [1] (who assumed a cylindrical-hemispherical droplet shape), Guigou-Carter, Villot and Horlaville [10] and Hopkins [11] (who assumed a paraboloidal droplet shape) and Griffin and Ballagh [12] (who consider cylindrical, cylindrical-hemispherical and paraboloidal droplet shapes). Yu and Hopkins [19] have also used this model to derive a force-time history for spherical and ellipsoidal droplet shapes (in addition to their more accurate empirical models). It is important to note that the droplet shapes used in these models do not reflect the actual droplet shape, but have been selected to provide a good match with measurements.

Recent detailed experimental studies have developed more accurate models of droplet impact forces. For Re $>200$, Gordillo, Sung and Cheng [17] showed that the force produced by a slowly moving liquid droplet (i.e. nearly spherical, and non-splashing) impacting on a horizontal, flat and rigid surface at times between impact and maximum force (the "initial impact regime") is indeed primarily caused by vertical to horizontal momentum transfer within the droplet. This momentum transfer occurs primarily within a small expanding volume of the droplet near to the contact area within which there are strong pressure gradients. Gordillo et al. analysed a large number of experimental measurements of droplet impact forces over a range of Reynolds numbers and observed that for Re $>200$, the dimensionless force $\tilde{F}_{1}(\tilde{t})$ collapsed onto a common curve which reaches a maximum value of $\tilde{F}_{1} \approx 0.85$ at $\tilde{t} \approx 0.18$. At times just after impact $(\tilde{t} \rightarrow 0)$, they found that $\tilde{F}_{1}(\tilde{t}) \sim \frac{3}{2} \sqrt{6} \tilde{t}^{0.5}$, in agreement with the asymptotic formulae proposed by Phillipi et al. [35]. After the maximum impact force, the strong pressure gradients within the droplet diminish in strength and the droplet enters the 'spreading regime' during which the force decays relatively slowly to zero.

Mitchell et al. [20] proposed the following semi-empirical model for the dimensionless force-time history for low-speed liquid droplet impacts on a flat horizontal surface:

$$
\tilde{F}_{1}(\tilde{t})=A_{M} \tilde{t}^{C_{M}} \exp \left\{-B_{M} \tilde{t}\right\},
$$


where $A_{M}=3.596, B_{M}=3.333$ and $C_{M}=0.500$. The coefficients $A_{M}$ and $B_{M}$ were determined so that the predicted time at which the maximum force occurred matched that measured and to ensure that the droplet impulse $\left(I=\int_{0}^{\infty} F_{1}(t) \mathrm{d} t\right)$ was equal to the momentum of the droplet just prior to impact. The coefficient $C_{M}$ was selected to give the correct asymptotic behaviour as $\tilde{t} \rightarrow 0$. Mitchell showed that this model provided a good fit to measured data - including closely matching the measured maximum force and accurately modelling the exponential slow decay during the spreading regime. In $\S 2.3$ we will also show that this profile provides a good match with our experimental data for small diameter droplets with relatively low impact velocities similar to those of a nominal intense rain drop impacting on surfaces at $0^{\circ}$ and $30^{\circ}$ angle of inclination. The dimensionless impact force profile defined by eq. (5) will be henceforth referred to as the intense rainfall impact force model.

Yu and Hopkins [19] also conducted a detailed experimental study measuring the force produced by $2 \mathrm{~mm}$ and $4.5 \mathrm{~mm}$ diameter water droplets impacting onto a horizontal glass surface. To calculate the impact force, they used a wavelet deconvolution method to process the response of the glass to the droplet impact as measured by a number of accelerometers. They deduced the following empirical relationship for the force time-history

$$
F_{1}(t)=C \exp \left\{-\frac{[\ln (A t)+\alpha]^{2}}{\beta^{2}}\right\}
$$

where $A=1000 \mathrm{~s}^{-1}, C=\exp \left\{U_{\perp} a_{C}+b_{C}\right\}, \alpha=U_{\perp} a_{\alpha}+b_{\alpha}, \beta=U_{\perp} a_{\beta}+b_{\beta}$ and the coefficients used in this model are given in table 1 .

Table 1. Coefficients for use in eq. 6

\begin{tabular}{|c|c|c|c|c|c|c|}
\hline Droplet diameter $[\mathrm{mm}]$ & $a_{C}\left[\mathrm{~m}^{-1} . \mathrm{s}\right]$ & $b_{C}[-]$ & $a_{\alpha}\left[\mathrm{m}^{-1} \cdot \mathrm{s}\right]$ & $b_{\alpha}[-]$ & $a_{\beta}\left[\mathrm{m}^{-1} . \mathrm{s}\right]$ & $b_{\beta}[-]$ \\
\hline 2 & 0.5088 & -5.001 & 0.1748 & 1.393 & -0.0727 & 1.3148 \\
\hline 4.5 & 0.4616 & -3.1084 & 0.3391 & 0.2307 & 0.0381 & 1.1176 \\
\hline
\end{tabular}

Yu and Hopkins' empirical formulae provide a good match with their experimental data. A minor deficiency with this model is that it does not produce the expected asymptotic behaviour as $\tilde{t} \rightarrow 0$ and nor does it satisfy the physical requirement that the impulse is equal to the droplet momentum just prior to impact for all cases.

The test method described in the ISO rainfall noise standard specifies that "the minimum slope of the test specimen is $5^{\circ}$ for roofs and $30^{\circ}$ for skylights. The slope used shall be the lowest that is feasible to assure water drainage". Thus, for the purposes of predicting the rainfall noise produced by a roof or skylight during tests conducted according to this standard, an impact force model which accounts for the effect of surface inclination is required. Yu and Hopkins [21] (following Zhang et al. [18]) proposed that the scaling given in eq. (4) used in conjunction with their empirical model given by eq. (6) can be used to calculate the impact force on inclined surfaces. This scaling is expected to work well for spherical droplets and, relative to a horizontal impact surface, will result in a reduction in the peak force magnitude and impulse and an increase in the time period between impact and maximum force. For ellipsoidal droplets, the effect of inclination on the impact force is similar, but more complicated due to the asymmetry of the impact process in these cases. Nevertheless, Yu and Hopkins [21] showed that there was generally good agreement between predictions made using this model and experimentally measured forces for $4.5 \mathrm{~mm}$ diameter water droplets impacting onto surfaces with inclination angles between $0^{\circ}$ and $60^{\circ}$ for impact velocities between $7.03 \mathrm{~m} \cdot \mathrm{s}^{-1}$ and $9.71 \mathrm{~m} \cdot \mathrm{s}^{-1}$. 
In addition to eq. (6), Yu and Hopkins [21] also proposed a second model for calculating droplet impact forces on an inclined surface based on that of Mitchell et al. [20]. The dimensionless force for this model is given by

$$
\tilde{F}_{1}(\tilde{t})=A_{Y} \tilde{t}^{C_{Y}} \exp \left\{-B_{Y} \tilde{t}^{\alpha}\right\},
$$

where $\alpha=\sec \theta$ and $C_{Y}=0.5$. The coefficients $A_{Y}$ and $B_{Y}$ were calculated to closely match the time at the peak force and the value of the peak force. For high-speed impacts of non-spherical droplets, $\mathrm{Yu}$ and Hopkins developed formulae for these coefficients using regression analysis of their extensive experimental data set. Predictions made using eq. (7) with these coefficients were shown to give good agreement with their experimentally measured force time-histories. Like eq. (6), eq. (7) does not necessarily produce the correct impulse. For impact on an inclined surface, the impulse should be equal to the momentum of the droplet in the direction perpendicular to the surface just prior to impact.

For heavy rain, where the droplets are relatively large, are ellipsoidal and have relatively high impact velocities, we propose using eq. (5) to model the non-dimensional impact force for droplets falling onto a flat, dry surface. The value of coefficients $A_{M}, B_{M}$ and $C_{M}$ are determined by matching the peak force and time at peak force with those predicted using the empirical model of Yu and Hopkins [21]. In addition, we require that the impulse is equal to the droplet momentum perpendicular to the surface just prior to impact. This yields $A_{M}=6.0413, B_{M}=4.5860$ and $C_{M}=0.5274$. The dimensionless impact force profile defined by eq. (5) with these parameters will be henceforth referred to as the heavy rainfall impact force model.

The Fourier transform of the force time-history produced by a single droplet impact is defined by

$$
\hat{F}_{1}(\omega)=\frac{1}{2 \pi} \int_{-\infty}^{\infty} F_{1}(t) \exp \{-\mathrm{i} \omega t\} \mathrm{d} t .
$$

For the impact force model defined by eq. (5), the Fourier transform evaluates as

$$
\hat{F}_{1}(\omega)=\frac{\rho_{w} D^{3} U_{\perp}}{2 \pi} \frac{A_{M} \Gamma\left(C_{M}+1\right)}{\left(B_{M}+\mathrm{i} \widetilde{\omega}\right)^{C_{M}+1}}
$$

where $\widetilde{\omega}=D \omega / U_{0}$ is a dimensionless frequency and $\Gamma(\cdot)$ is the gamma function.

Figure 2 plots the spectrum predicted by the intense and heavy rainfall impact force models for droplet impacts on a horizontal surface and compares it with numerical calculations of the spectra of $\mathrm{Yu}$ and Hopkins' empirical model and the paraboloidal droplet impact model. The paraboloidal model is included as it has been commonly used in previous rainfall noise prediction methods (e.g. [10]-[12], [16]). Note that the prediction using Yu and Hopkins' model for the $5 \mathrm{~mm}$ heavy rainfall droplet has been scaled assuming the non-dimensional force is identical to that produced by a $4.5 \mathrm{~mm}$ diameter droplet. There is good agreement (within approximately $1 \mathrm{~dB}$ at all frequencies) between the proposed heavy and intense rainfall impact force models and the empirical model of $\mathrm{Yu}$ and Hopkins. However, there are significant discrepancies between those models and the paraboloidal model. This demonstrates that the accuracy of many of the previously published rain noise prediction methods could be improved by incorporating a more accurate droplet impact force model. 

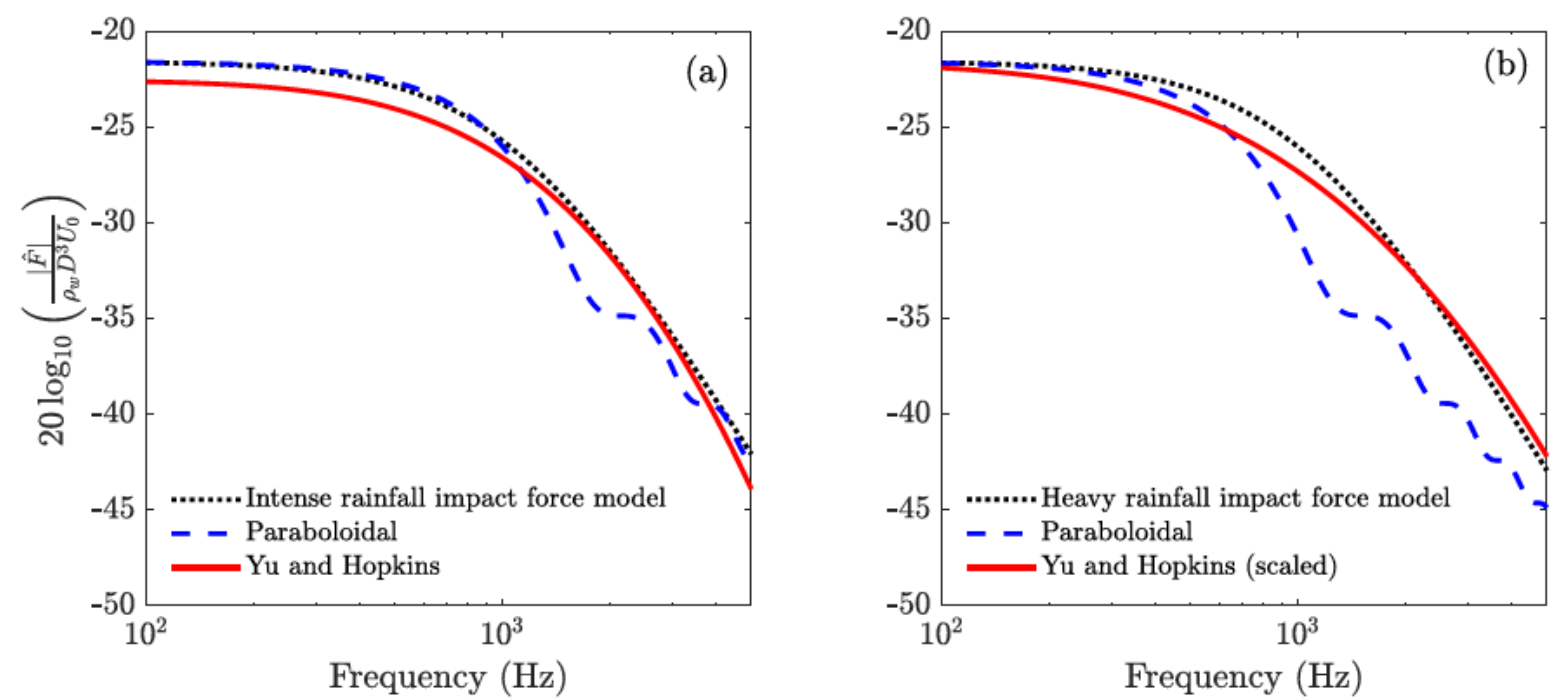

Figure 2. Predicted single droplet impact force spectrum. (a) a $2 \mathrm{~mm}$ diameter droplet impacting on a horizontal surface at $4 \mathrm{~m} \cdot \mathrm{s}^{-1}$; (b) a $5 \mathrm{~mm}$ diameter droplet impacting on a horizontal surface at $7 \mathrm{~m} \cdot \mathrm{s}^{-1}$.

\subsection{Comparison between predicted and measured impact forces}

In the remainder of this section we describe an experimental study investigating the impact force produced by liquid droplets on horizontal and inclined dry surfaces. To the best of our knowledge, forces produced by droplets impacting on an inclined surface have only been reported previously by Zhang et al. [18] and Yu and Hopkins [21]. In §2.3.1. a detailed experimental study is presented which investigates the impact force time-history produced by droplets falling from up to $1 \mathrm{~m}$ onto a surface. The goal is to assess the accuracy of the intense rainfall droplet impact force model. In $\S 2.3 .2$, an experimental investigation of the impact force produced by large droplets falling from $3.5 \mathrm{~m}$ onto a surface is described. The goal is to assess the accuracy of the heavy rainfall droplet impact force model. Each investigation uses a different experimental setup and thus they are reported separately.

\subsubsection{Low-speed droplet impact force measurement method}

The general experimental method used to measure the impact force of low-speed droplets follows that described by Gordillo et al. [17] and the experimental setup is shown in figure 3. Force measurements were made using a highly sensitive piezoelectric pressure sensor (PCB 106B51) connected to a National Instruments 9205 CompactDAQ System via a signal conditioner (PCB 480C02). The signal was sampled at $250 \mathrm{kHz}$. Gordillo et al. [17] reported that the measurement of impact forces using piezoelectric transducers is confounded by "the presence of strong resonant ringing" and "abnormal slow decay", which they attributed to an electrical interaction of the polar liquid with the piezoelectric sensor. Other investigators (e.g. refs. [23], [24], [25], [26] and [27]) have reported similar problems. Gordillo et al. mitigated these issues by targeting the impinging drops slightly off the centre of the force sensor, which significantly reduced resonant ringing. Furthermore, they used droplets of nonpolar silicone oil to reduce the slow decay of the measured force signal. Thus, in the experiments reported here, we investigated the force produced by the impact of both water droplets and droplets of different silicone oils (with kinematic viscosities between $5 \mathrm{cSt}$ and 1,000 cSt). These droplets were generated using a syringe pump. 


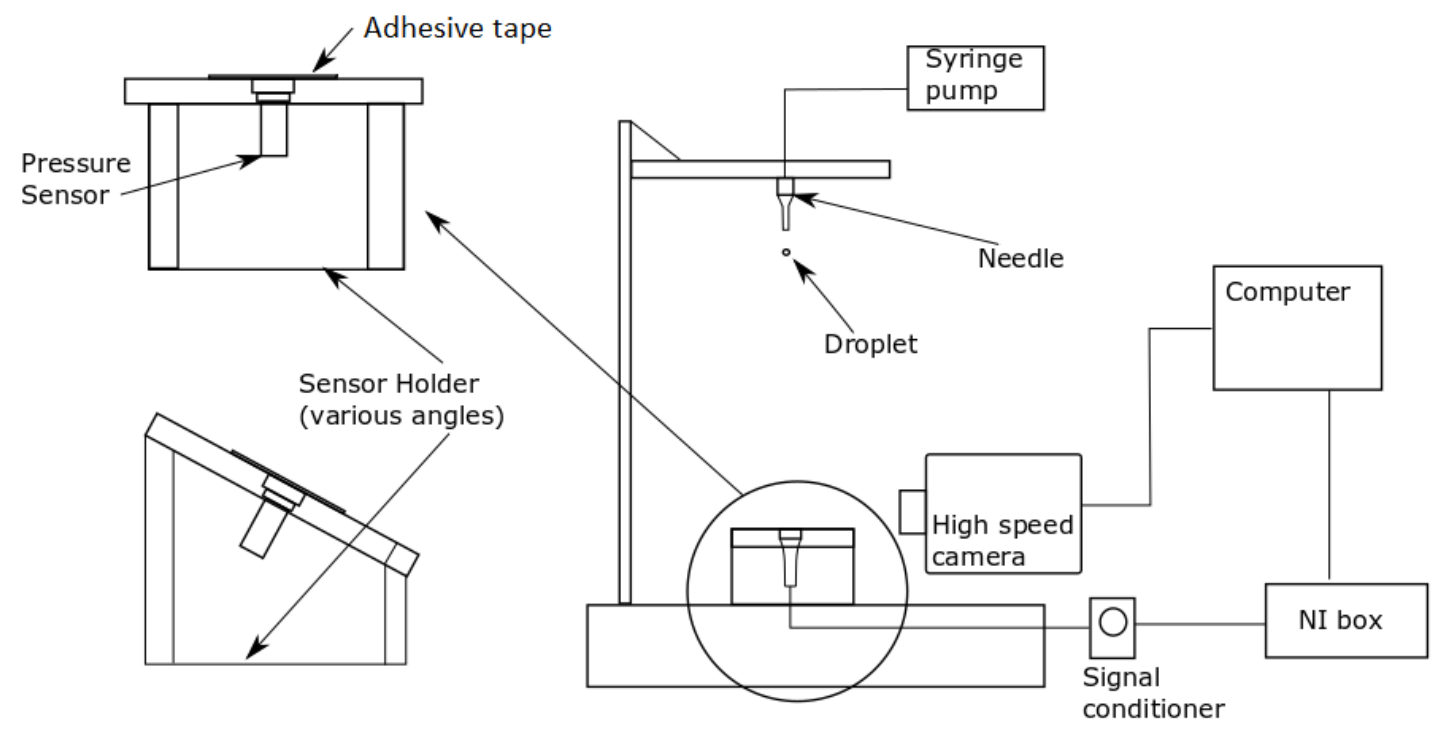

Figure 3. Experimental setup to measure low-speed droplet impact force

When the droplets impacted the piezoelectric sensor directly, in the case of water droplets and low viscosity silicone oils an "abnormal slow decay" was observed, the size of which was dependent on the liquid, and which we believe is identical to the phenomenon reported by Gordillo et al. [17]. Following advice received via a personal communication with the lead author of ref. [17], we reduced this phenomenon by applying one or more layers of plastic adhesive tape over the sensing surface of the transducer. The pressure sensor was then calibrated using an impact hammer (PCB 086E90), and the calibration was also verified by comparing the measured impulse with the momentum of impinging drops, as described in Appendix A. The sensor has a rise time of $10 \mu \mathrm{s}$, and a force resolution of 0.3 $\mathrm{mN}$. It has a resonant frequency of approximately $50 \mathrm{kHz}$. The recorded force time-history was smoothed using a 5-point moving average which acted as a low-pass filter to remove this resonance (see figure 4).

All experiments were performed in a laboratory with ambient temperature $21 \pm 1{ }^{\circ} \mathrm{C}$ and relative humidity 50-75\%. Table 2 shows the range of parameters used in the experiments. Various drop heights up to $1 \mathrm{~m}$ were used to generate impact velocities of 2-3 m.s. droplets. The drop velocity and size were determined from high-speed camera footage. Camera footage of the droplet impacts was captured using a FastCAM SA5 camera with a frame rate of 10,000 frames per second, a shutter speed of $18.2 \mu \mathrm{s}$, and with a resolution of $896 \times 848$ pixels. The length of each pixel in a photograph was determined by photographing a $4 \mathrm{~mm}$ square calibration tile located close to the droplet impact location prior to conducting a series of experiments. The sensor was either mounted with the impact surface horizontal or inclined at an angle of $20^{\circ}, 30^{\circ}$ or $40^{\circ}$ to the horizontal. The sensor has a $15 \mathrm{~mm}$ diameter circular sensing surface and so droplets were contained fully on the sensor at low velocities. However, at higher velocities it was difficult to aim drops to ensure the drop impacts were contained on the sensor. This was overcome by conducting multiple trials and verifying drop aim with camera footage. 

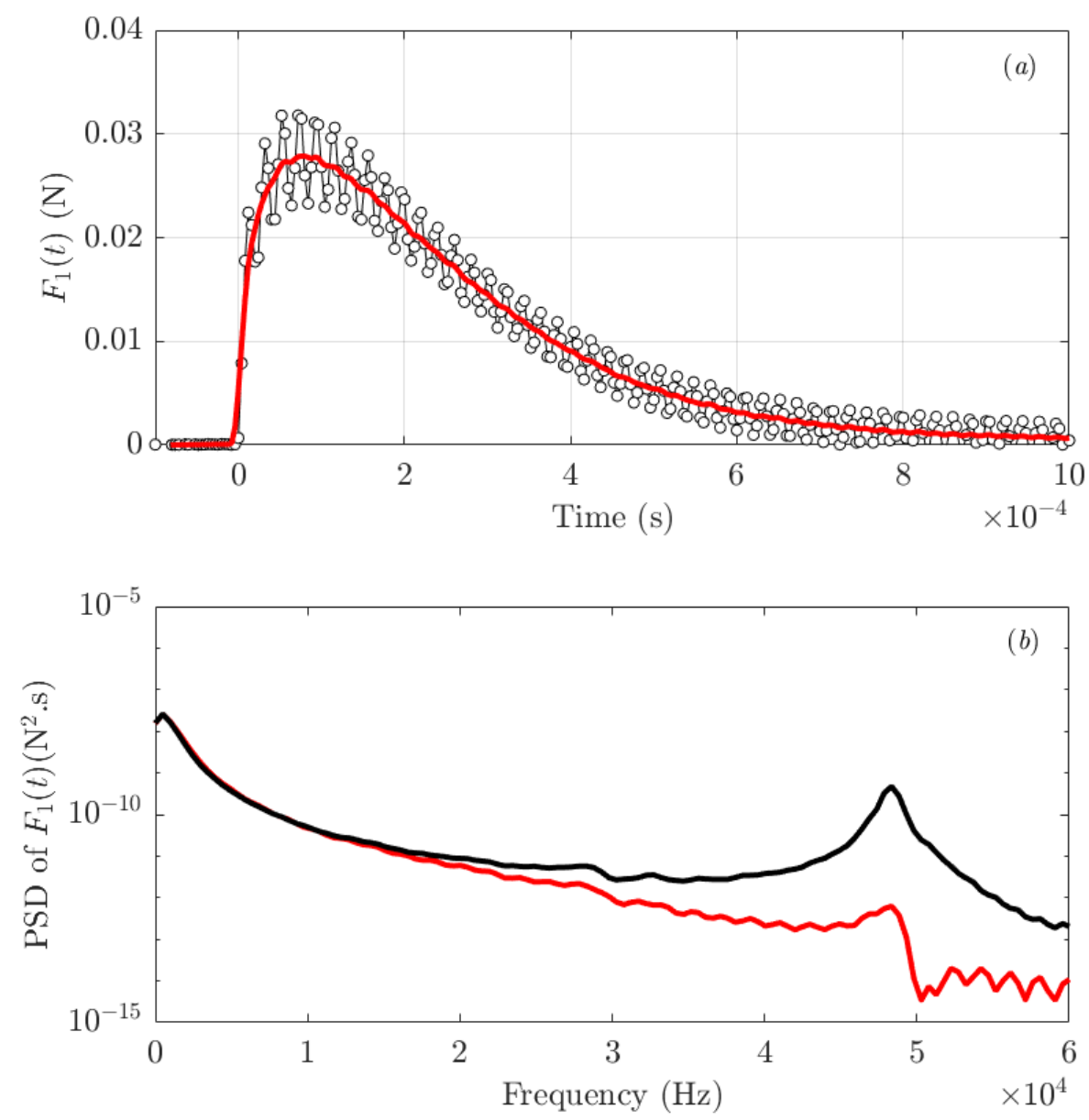

Figure 4. (a) Measured force profile for impact of a silicone oil droplet (with kinematic viscosity of $100 \mathrm{cSt}$ ) falling from a drop height $H=50 \mathrm{~cm}$. The raw signal is shown in black whilst the signal after filtering using a 5-point moving average was applied is shown in red. (b) Frequency spectrum before (black) and after (red) moving average was applied.

Table 2. Summary of low-speed droplet impact experiments

\begin{tabular}{|l|l|l|l|l|}
\hline Fluid & $D(\mathrm{~mm})$ & $U_{0}(\mathrm{~m} / \mathrm{s})$ & $H(\mathrm{~cm})$ & $\theta\left(^{\circ}\right)$ \\
\hline Water & $2.6-4.5$ & $2-4$ & $25-100$ & 0,30 \\
\hline $\begin{array}{l}\text { Silicone Oil } \\
5-1000 \mathrm{cSt}\end{array}$ & $1.9 \pm 0.1$ & $2-3$ & $25-50$ & $0,20,30,40$ \\
\hline
\end{tabular}

The droplet shape was assessed using the aspect ratio $e$ which is defined as the ratio of the droplet height $\left(D_{v}\right)$ to the width $\left(D_{h}\right)$ i.e. $e=D_{v} / D_{h}$. The droplet diameter was calculated assuming an ellipsoidal shape which gives $D=\left(D_{h}^{2} D_{v}\right)^{1 / 3}$. As expected for silicone oil droplets at velocities $2<U_{0}<3 \mathrm{~m} / \mathrm{s}$ and with nominal diameters $D \approx 1.9 \mathrm{~mm}$, the droplets were approximately spherical $(0.93 \leq e \leq 1.0)$. For water droplets at velocities $2<U_{0}<4 \mathrm{~m} / \mathrm{s}$ and with nominal diameters $D \approx 2.6 \mathrm{~mm}$ the droplets were also approximately spherical, $0.9 \leq e \leq 1.0$. For larger water droplets, $D \approx 4.1 \mathrm{~mm}$ at velocities of $2<U_{0}<4 \mathrm{~m} / \mathrm{s}$ the droplets were found to be oblate or prolate, depending 
on the drop height, due to oscillations as the droplet fell. Example droplet shapes are shown in figure 5 (a) $-(\mathrm{d})$.

$$
\begin{aligned}
& e=0.96 \\
& D=1.85 \mathrm{~mm}
\end{aligned}
$$

Silicone Oil, 5cSt

$$
D_{h}=1.87
$$

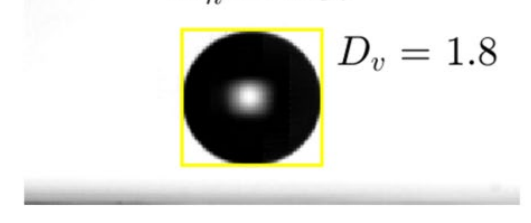

$$
\begin{aligned}
& e=0.88 \\
& D=4.06 \mathrm{~mm} \\
& \text { Water }
\end{aligned}
$$

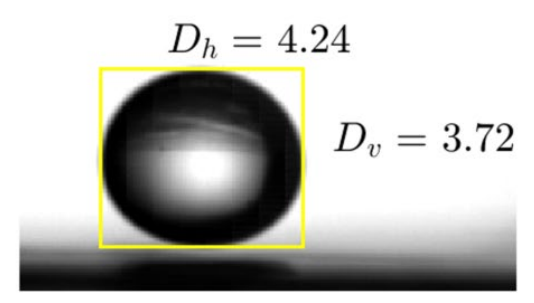

$(c)$

$$
\begin{aligned}
& e=0.97 \\
& D=2.65 \mathrm{~mm}
\end{aligned}
$$

Water

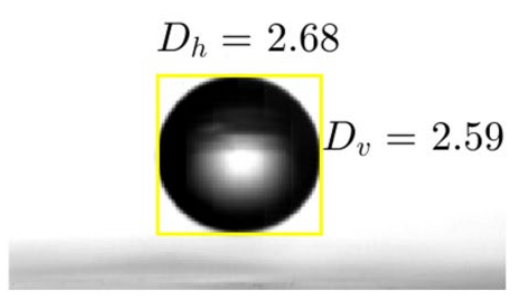

$$
\begin{aligned}
& e=1.08 \\
& D=4.05 \mathrm{~mm} \\
& \text { Water }
\end{aligned}
$$

$$
D_{h}=3.96
$$

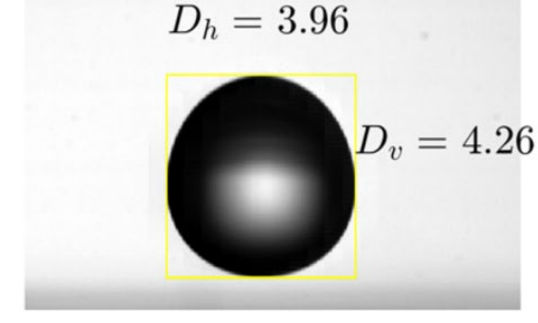

Figure 5. Different drop shapes: (a) spherical, 5 cSt silicone oil (b) spherical, water (c) oblate, water and (d) prolate, water. $D_{h}$ and $D_{v}$ are expressed in $\mathrm{mm}$.

Figure 6 plots the impact force versus time data produced by two silicone oil droplets of similar size, and with kinematic viscosity of $5 \mathrm{cSt}$, impacting on a horizontal surface at different velocities. The measured force profiles are compared with predictions made using the formula proposed by Mitchell et al. [20] (eq. 5) showing excellent agreement. 

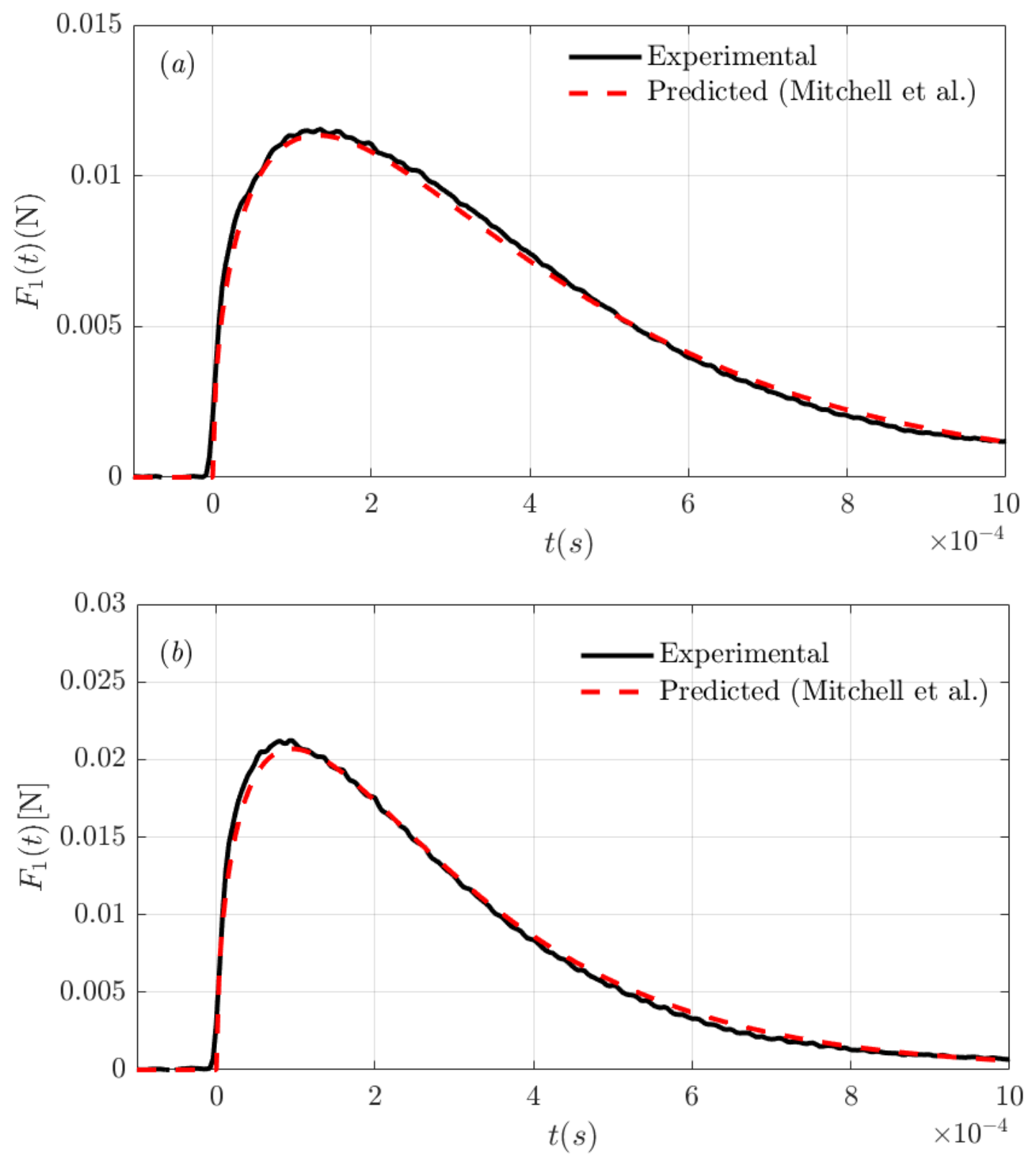

Figure 6. Impact force versus time produced by a two similar size silicone oil droplets (with kinematic viscosity of $5 \mathrm{cSt}$ ) impacting on a horizontal surface at different velocities: (a) $D=1.85 \mathrm{~mm}$ and $U_{0}=$ $2.05 \mathrm{~m} / \mathrm{s}(H=25 \mathrm{~cm}, \operatorname{Re}=773, e=0.96)$; (b) $D=1.82 \mathrm{~mm}$ and $U_{0}=2.86 \mathrm{~m} / \mathrm{s}(H=50 \mathrm{~cm}, \operatorname{Re}=$ $1044, e=0.93)$. The experimentally measured force is compared with a prediction made using the formula proposed by Mitchell et al. [20] (eq. 5).

Figure 7 plots the impact force versus time produced by a $2.66 \mathrm{~mm}$ diameter water droplet impacting on a horizontal, dry surface at $U_{0}=4.04 \mathrm{~m} / \mathrm{s}$. The experimentally measured force is compared with predictions made using the formula proposed by Mitchell et al. (eq. 5), which is valid for spherical droplets and the empirical formula of Yu and Hopkins (eq. 6) for a $2 \mathrm{~mm}$ droplet (scaled to account for the difference in droplet diameter). There is very good agreement between the measurements and the prediction made using the model of Mitchell et al. The agreement between the measured peak force and 
that predicted using the empirical model of $\mathrm{Yu}$ and Hopkins is in reasonable agreement, although there is some error in the predicted impulse and time at which peak impact force occurs for this case.

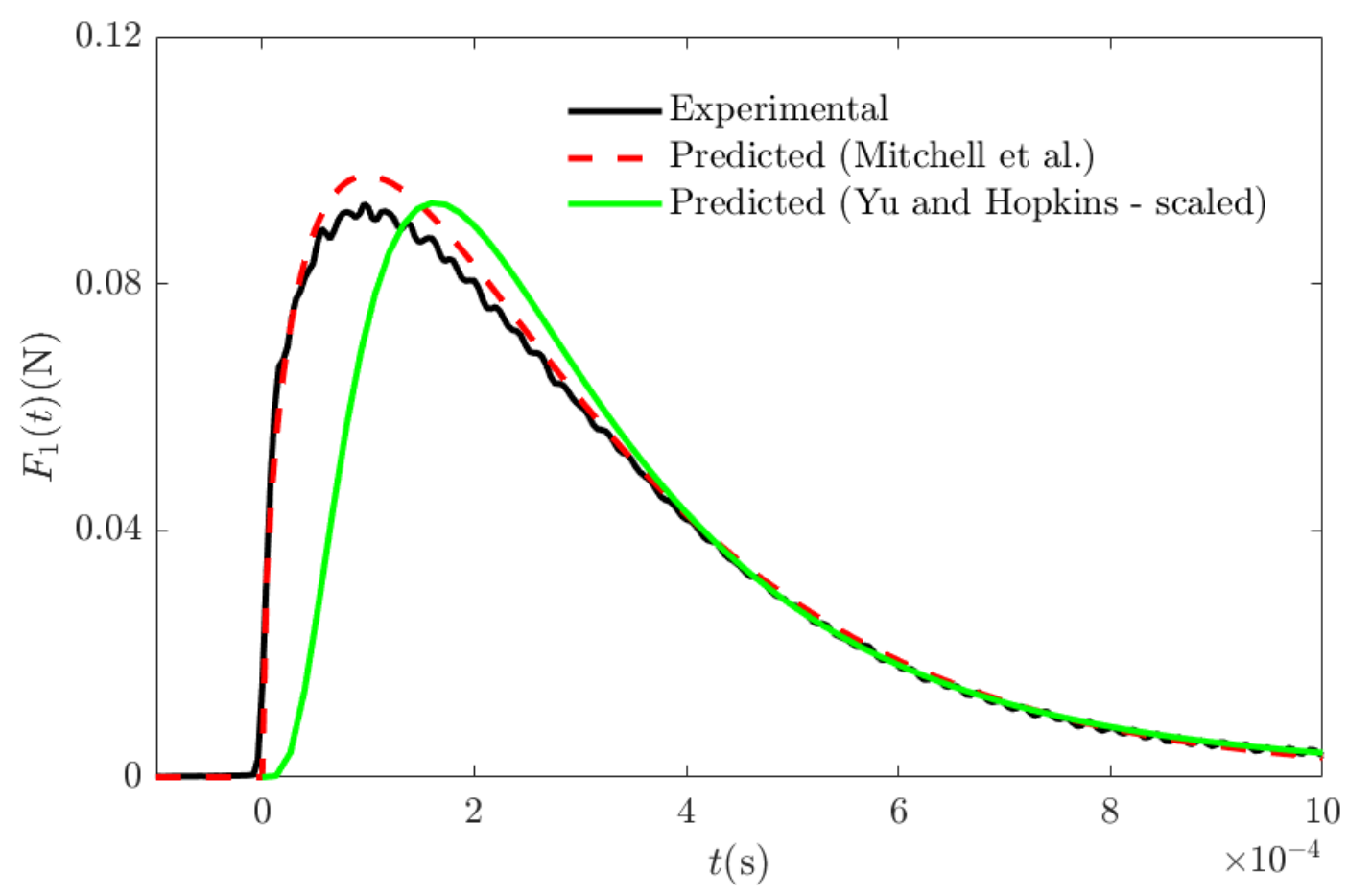

Figure 7. Impact force versus time produced by a $D=2.66 \mathrm{~mm}$ diameter water droplet impacting on a horizontal surface at $U_{0}=4.04 \mathrm{~m} / \mathrm{s}(H=100 \mathrm{~cm}, \operatorname{Re}=10733, e=0.97)$. The experimentally measured force is compared with predictions.

Figure 8 plots the impact force time-history produced by two $\approx 4 \mathrm{~mm}$ nominal diameter, non-spherical water droplets impacting on a horizontal surface at $\approx 4 \mathrm{~m} / \mathrm{s}$. Each droplet was highly non-spherical just before impact with the results plotted in the figure 8 (a) corresponding to a droplet which was oblate $(e$ $=0.88)$ just before impact and the results in figure $8(\mathrm{~b})$ corresponding to a droplet which was prolate $(e=1.08)$ just before impact. The measurements are compared with predictions made using the models of Mitchell et al. (eq. 5) and of $\mathrm{Yu}$ and Hopkins (eq. 6 for a $4.5 \mathrm{~mm}$ droplet, scaled to account for differences in the droplet diameter). The measurements are typical and show that oblate droplets produce a slightly more impulsive force with a higher peak force which occurs sooner after impact relative to the predicted force profile for a spherical droplet. The measured force profile for the prolate droplet shown in figure 8 (b) has a lower peak force amplitude than that predicted for the spherical droplet, although the time at peak force is somewhat smaller than that predicted for a spherical droplet. 

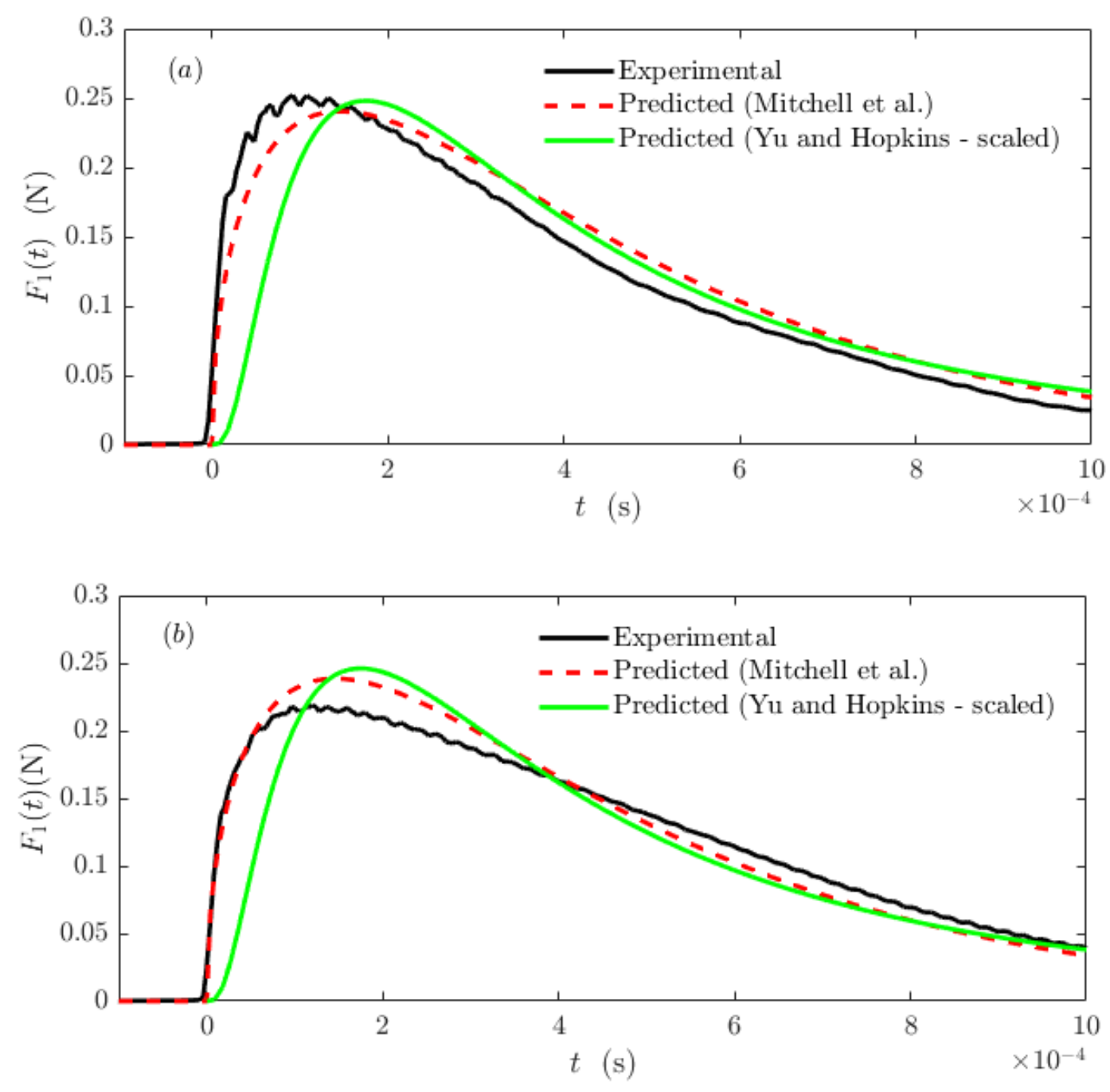

Figure 8. Impact force versus time produced by a two similar size water droplets impacting on a horizontal surface at similar velocities but with different shapes just before impact: (a) oblate drop, $D=4.06 \mathrm{~mm}, U_{0}=4.16 \mathrm{~m} / \mathrm{s}, H=100 \mathrm{~cm}, \operatorname{Re}=16857, e=0.88$; and (b) prolate drop, $D=4.05 \mathrm{~mm}$, $U_{0}=4.15 \mathrm{~m} / \mathrm{s}, H=100 \mathrm{~cm}, \operatorname{Re}=16788, e=1.08$. The experimentally measured force is compared with predictions.

Figures 9 and 10 plot the impact force-time history produced by spherical droplets impacting on a surface at low speeds inclined at $30^{\circ}$ to the horizontal. The experimentally measured force is compared with predictions made using the formula proposed by Mitchell et al. (eq. 5) scaled using the droplet impact velocity perpendicular to the impact surface. This scaling produces predictions which are in good agreement with measurements and validates the accuracy of this scaling method for spherical droplets.

The results presented in this section are representative of our other experiments and show that the droplet impact force for low speed, spherical droplets can be predicted with good accuracy using the model of Mitchell et al. (eq. 5). For a nominal intense rain droplet with a diameter of $2 \mathrm{~mm}$ and an 
impact velocity of $4 \mathrm{~m} \cdot \mathrm{s}^{-1}$ this model corresponds to the intense rainfall impact model. For non-spherical droplets, this equation is less accurate and thus a different model is required.

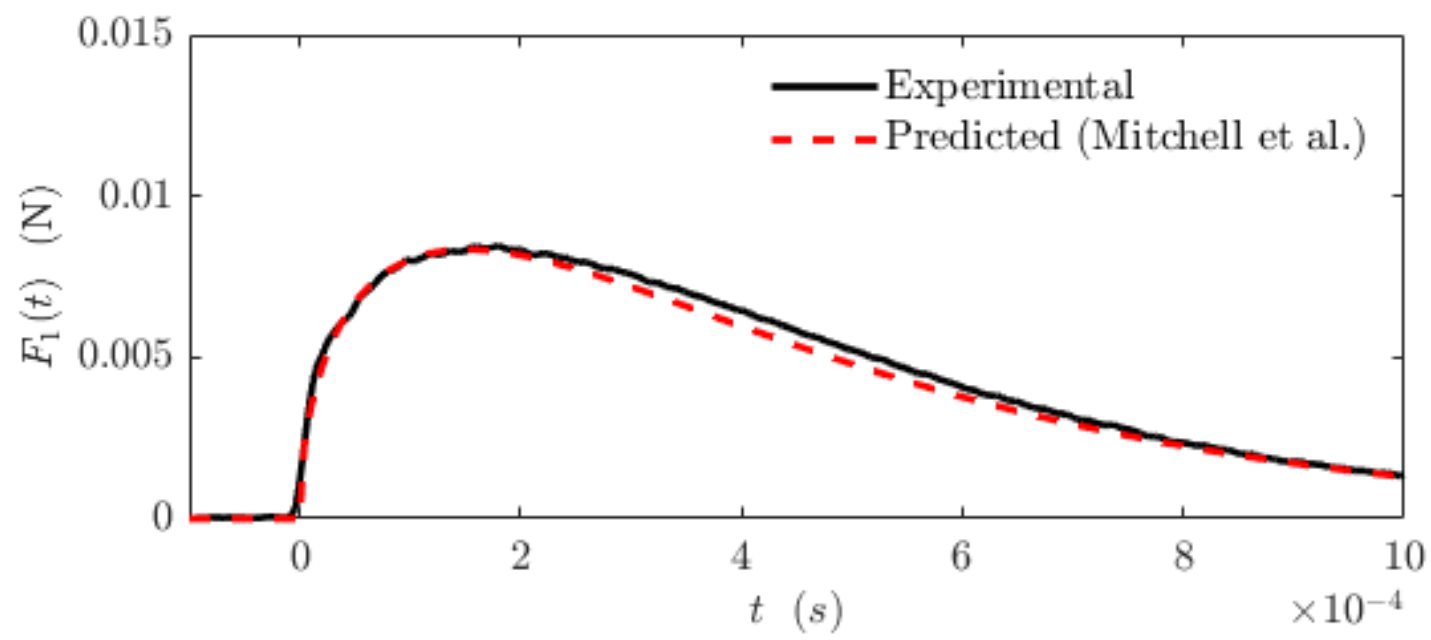

Figure 9. Measured and predicted impact force versus time produced by a $5 \mathrm{cSt}$ silicone oil droplet impacting on a surface inclined at $30^{\circ}$ to the horizontal: $D=1.83 \mathrm{~mm}, U_{0}=2.09 \mathrm{~m} / \mathrm{s}, H=25 \mathrm{~cm}$, $\operatorname{Re}=765$ and $e=0.96$.

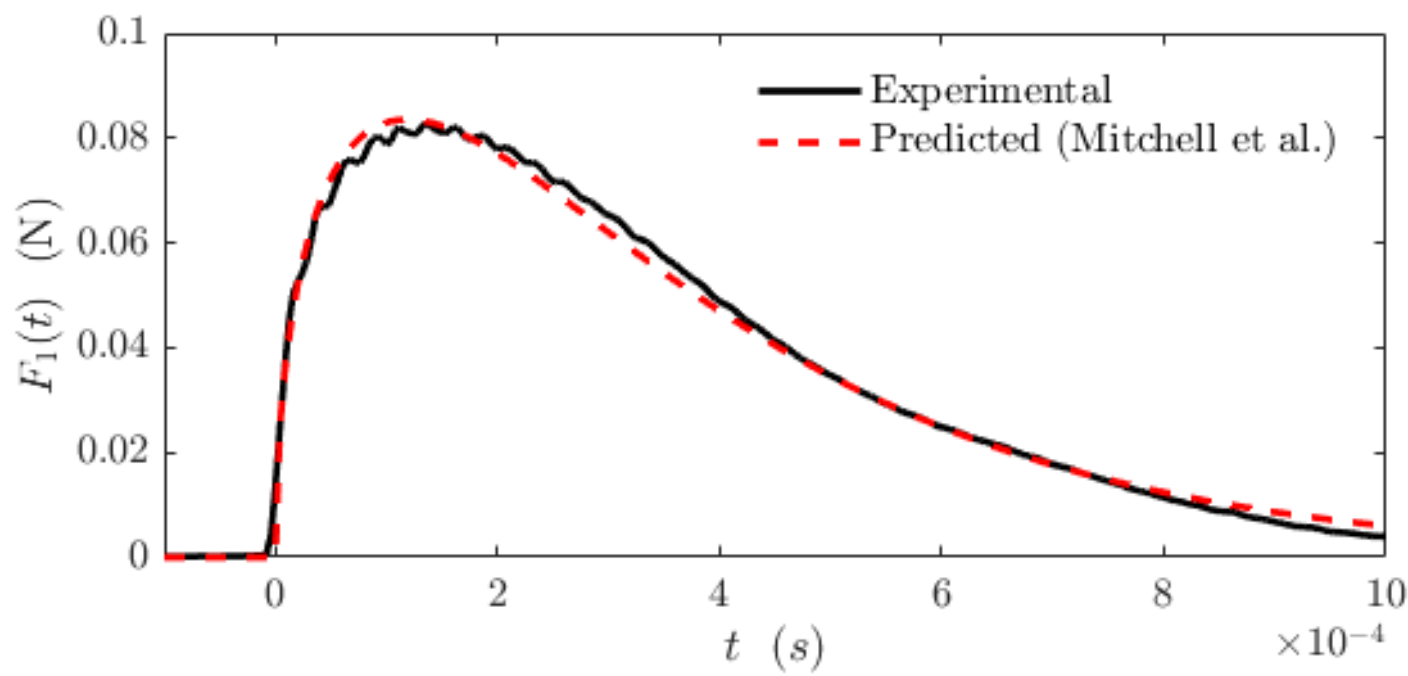

Figure 10. Measured and predicted impact force versus time produced by a spherical water droplet impacting on a surface inclined at $30^{\circ}$ to the horizontal: $D=2.81 \mathrm{~mm}, U_{0}=4.09 \mathrm{~m} / \mathrm{s}, H=100 \mathrm{~cm}$, $\operatorname{Re}=11875, e=0.96$.

\subsubsection{High-speed droplet impact force measurement method}

Experiments were also conducted for large water droplets falling from a height of $3.5 \mathrm{~m}$. Droplets were generated using the drip tray used for the rain noise tests described in $\S 4$. The size of these droplets was assessed using a method described in $\S 4$ and was found to be very consistent, with a mean diameter of $6.04 \mathrm{~mm}$. Note that because of their size and velocity, each droplet is non-spherical at impact. A photograph of a single droplet just prior to impact is shown in figure 11. The impact velocity calculated using eq. (1) for a droplet with a nominal diameter of $6.04 \mathrm{~mm}$ is $7.23 \mathrm{~m} / \mathrm{s}$. 


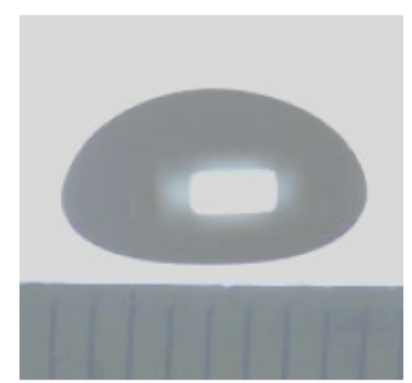

Figure 11. Photograph of a high-speed droplet just before impact. There is a $1 \mathrm{~mm}$ space between the ruler scale markings.

The impact force was measured using a force transducer (PCB 208 B02) glued to a large mass. A $3.1 \mathrm{~mm}$ thick, $28 \mathrm{~mm}$ diameter circular disc made from carbon fibre was mounted onto the top of the transducer onto which droplets impacted. The transducer was mounted at an angle of inclination of either $5^{\circ}$ or $30^{\circ}$ corresponding to the angle of inclination of the rooves tested in $\S 4$. This system produced a resonance at approximately $16 \mathrm{kHz}$, which made plotting a force time-history problematic. Thus, in this section we instead present measured impact force spectra for frequencies between $50 \mathrm{~Hz}$ and $5 \mathrm{kHz}$, which corresponds to the range of interest for rain noise measurements made to the ISO rainfall noise standard. This force measurement system was calibrated by matching the measured impulse to the expected change in momentum.

Figure 12 plots the measured force spectra for the $5^{\circ}$ and $30^{\circ}$ inclination angle cases, nondimensionalised by $\rho_{\boldsymbol{w}}, D$ and $U_{\perp}$, and compares this measured data with the heavy rainfall impact force model (eq. 5) and $\mathrm{Yu}$ and Hopkins empirical model (eq. 6 for a $4.5 \mathrm{~mm}$ droplet, scaled to account for differences in the droplet diameter) for the two cases. There is reasonable agreement between both nondimensionalised measured spectra and also between these spectra and the theoretical models.

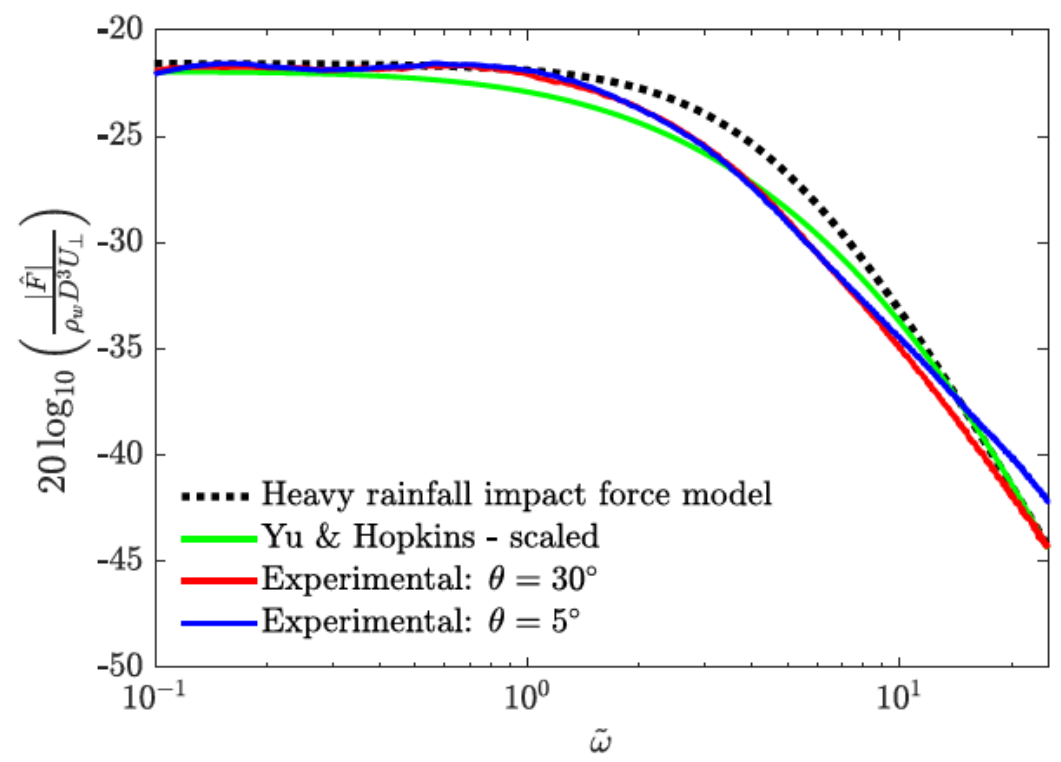

Figure 12. Non-dimensional force spectra produced by a $6.04 \mathrm{~mm}$ drop impacting on a surface inclined at $5^{\circ}$ (solid red) and $30^{\circ}$ (solid blue) and compared with the heavy rainfall impact force model (black dotted) and Yu and Hopkins' empirical model (green). 


\section{Theoretical model for the sound power produced by droplet impacts on a flat panel.}

In this section, theoretical models for calculating the sound power produced by rain on a flat, inclined panel due to the impact forces produced by artificial rainfall are presented. The roof is modelled as a thin, isotropic plate with mass-per-unit-area $m$, stiffness $D$ and damping factor $\eta$, for which the equilibrium position of the plate mid-surface lies in the $z=0$ plane. Rainfall is assumed to consist of a series of identical droplets falling from the holes in the underside of a tray that complies with the specifications listed in the ISO rainfall standard. We assume that a sequence of identical droplets falling from one hole in the tray impact on the plate at a point with a constant period $T_{1}$ between impacts. This produces a total periodic impact force $F(t)$ in the negative $z$ direction, which is a sum of the force produced by each identical droplet impact i.e.

$$
F(t)=\sum_{n=-\infty}^{\infty} F_{1}\left(t+n T_{1}\right),
$$

where $F_{1}\left(t+n T_{1}\right)$ is the force produced by the $n^{\text {th }}$ droplet impact. Making use of Poisson's summation formula yields

$$
F(t)=\sum_{n=-\infty}^{\infty} F^{(n)} \exp \left\{\mathrm{i} \omega_{n} t\right\}
$$

where $F^{(n)}$ is the amplitude of the $n^{\text {th }}$ Fourier harmonic which is defined as $F^{(n)}=2 \pi \widehat{F}_{1}\left(\omega_{n}\right) / T_{1}$, $\omega_{n}=2 \pi n / T_{1}$ and $\hat{F}_{1}(\omega)$ is the Fourier transform of the single $(n=0)$ droplet impact force which is defined by eq. (8).

Petersson [16] suggests that the effect of surface compliance on the impact force may be neglected if the driving point impedance of the plate $Z_{\mathrm{dp}} \gg Z_{\text {flow }}$, where for a thin, isotropic plate $Z_{\mathrm{dp}}=8 \sqrt{D m}$ (see Hopkins [11]) and $Z_{\text {flow }}=\frac{\pi}{4} \rho_{w} D^{2} U_{0}$ is the 'flow impedance' of the droplet. A lightweight steel roof comprising $0.5 \mathrm{~mm}$ thick flat steel plate has a driving point impedance of $Z_{\mathrm{dp}}=26.7 \mathrm{~kg} \cdot \mathrm{s}^{-1}$, whilst a $6 \mathrm{~mm}$ thick glass plate, which might be suitable as a skylight, has a driving point impedance of $1107 \mathrm{~kg} . \mathrm{s}^{-1}$. For a heavy rain droplet with a diameter of $5 \mathrm{~mm}$ impacting at $7 \mathrm{~m} / \mathrm{s}$, $Z_{\text {flow }}=0.137 \mathrm{~kg} \cdot \mathrm{s}^{-1}$, whilst for an intense rain droplet with a diameter of $2 \mathrm{~mm}$ impacting at $4 \mathrm{~m} / \mathrm{s}$, $Z_{\text {flow }}=0.0126 \mathrm{~kg} \cdot \mathrm{s}^{-1}$. Thus, for typical rooves and skylights, the effect of surface compliance on the force applied by the droplets can be neglected.

As mentioned previously, $\mathrm{Yu}$ and Hopkins [19] have shown that a thin film of water on the impact surface may affect the impact force. We do not account for this effect here, although we note that in our experience during testing on surfaces at low angles of incidence, the impact surface remained slightly wet such that small puddles sometimes formed close to some droplet impact locations. This would undoubtedly have affected the impact force at these locations. Care therefore does need to be taken to ensure that the roof is inclined at a suitable angle to prevent water pooling.

We assume that the roof response is independent of excitation location and thus, for a tray with $N$ holes, the power input to the plate by the rain at frequency $f_{n}=\left|\omega_{n}\right| /(2 \pi)\left(f_{n}>0\right)$ is approximated by 


$$
W_{\mathrm{in}}^{(n)} \approx \frac{2 N\left|F^{(n)}\right|^{2}}{Z_{\mathrm{dp}}}
$$

Note that because $F^{(n)}$ is the amplitude coefficient of a double-sided Fourier series, $\left|F^{(n)}\right|^{2}$ is equal to the mean-square force at frequency $f_{n}$.

The rainfall rate falling from a tray of horizontal cross-sectional area $A_{\text {tray }}$ is denoted $\dot{H}$. The volume flow rate of water from each hole is $\dot{V}=A_{\text {tray }} \dot{H} / N$. Because each droplet has a volume $V=\pi D^{3} / 6$, the period of time between droplets is thus

$$
T_{1}=\frac{V}{\dot{V}}=\frac{\pi N D^{3}}{6 A_{\text {tray }} \dot{H}} .
$$

The time-average sound power radiated from a finite panel excited by rainfall at frequency $f_{n}$ is denoted $\bar{\Pi}^{(n)}$. Three different methods for calculating $\bar{\Pi}^{(n)}$ are described in $\S \S 3.1-3$.

The sound power in the $m$ th one-third octave band with lower, and upper band frequencies $f_{l}^{(m)}$ and $f_{u}^{(m)}$ is

$$
\bar{\Pi}_{1 / 3}^{(m)}=\sum_{n=m_{l}}^{m_{u}} \bar{\Pi}^{(n)}
$$

where $m_{l}=\left\lceil f_{l}^{(m)} T_{1}\right\rceil$ and $m_{u}=\left\lfloor f_{u}^{(m)} T_{1}\right\rfloor$.

The one-third octave band sound power level is defined as

$$
L_{w}=10 \log _{10}\left(\frac{\bar{\Pi}_{1 / 3}^{(m)}}{\bar{\Pi}_{\mathrm{ref}}}\right), \quad \bar{\Pi}_{\mathrm{ref}}=10^{-12} \mathrm{~W} .
$$

\subsection{Radiation model 1}

Davy et al. [36] (see also Davy [37] and Davy et al. [38]) shows that the sound power radiated from a finite panel excited at frequency $f_{n}$ by a point force is given by

$$
\bar{\Pi}^{(n)}\left(\omega_{n}\right) \approx \frac{\rho_{0} c_{0} \sigma_{r} W_{\mathrm{in}}^{(n)}}{m \omega_{n} \eta} R, \quad R=\left\{\begin{array}{cc}
1+\frac{4 f_{n} \eta}{\pi f_{c} \sigma_{r}}, & f_{n}<f_{c} ; \\
1, & f>f_{c} .
\end{array}\right.
$$

In eq. (16), $\sigma_{r}$ is taken to be the 'azimuthal average resonant radiation efficiency' of the plate which is defined in ref. [36] and $f_{c}=\frac{c_{0}^{2}}{2 \pi} \sqrt{\frac{m}{D}}$ is the critical frequency of the plate. Eq. (16) is equivalent to the sound power formulae presented in Griffin and Ballagh [12] for a given force input, except that they incorporate a different expression for the radiation efficiency. Both methods incorporate the sound power radiated from what are commonly referred to as the 'resonant' and 'non-resonant' vibration fields. Similar methods for calculating the sound power radiated from rainfall on a roof have been implemented by Guigou-Carter et al. [10] and Hopkins [11]. 


\subsection{Radiation model 2}

The model presented in $\S 3.1$ is suitable for isotropic, homogeneous panels at frequencies high enough that modal effects are not important. Here we present an alternative model which accounts for modal effects due to the finite size of the test specimen. The approach is restricted to a thin panel. The panel is rectangular in shape occupying $0 \leq x \leq L_{x}$ and $0 \leq y \leq L_{y}$ with thickness $L_{z}$ and is mounted in an infinite baffle which occupies the $z=0$ plane. Rain impinges on the upper surface of the panel which excites it and causes sound to radiate from both the top and bottom surfaces.

A panel which is excited by a periodic point force of the form given by eq. (11) will produce a transverse displacement $w(x, y, t)$ which is also periodic and can therefore be expressed as a Fourier series i.e.

$$
w(x, y, t)=\sum_{n=-\infty}^{\infty} \widehat{w}\left(x, y, \omega_{n}\right) \exp \left\{\mathrm{i} \omega_{n} t\right\} .
$$

The equation of motion for a thin, isotropic, homogeneous plate subjected to a periodic point force $F^{(n)} \exp \left\{\mathrm{i} \omega_{n} t\right\}$ applied at $x=x_{0}$ and $y=y_{0}$ is given by

$$
\left[D(1+\mathrm{i} \eta)\left(\frac{\partial^{2}}{\partial x^{2}}+\frac{\partial^{2}}{\partial y^{2}}\right)^{2}-m \omega_{n}^{2}\right] \widehat{w}\left(x, y, \omega_{n}\right)=F^{(n)} \delta\left(x-x_{0}\right) \delta\left(y-y_{0}\right) .
$$

Note that the effect of fluid loading on the panel response is neglected in this analysis. This is justified as this effect is negligibly small in comparison to the loading due to the rainfall.

The displacement of the rectangular panel in the $z$-direction can be decomposed into a sum of mode shape functions, $\phi_{q}$, each with amplitude $a_{q}$, i.e.

$$
\widehat{w}\left(x, y, \omega_{n}\right)=\sum_{q=1}^{\infty} a_{q}\left(\omega_{n}\right) \phi_{q}(x, y) .
$$

For a thin, rectangular plate with pinned edges, the modal shape functions are given by (see ref. [39])

$$
\phi_{q}(x, y)=\sin \left(\frac{q_{x} \pi x}{L_{\mathrm{x}}}\right) \sin \left(\frac{q_{y} \pi y}{L_{\mathrm{y}}}\right)
$$

where $q_{x}$ and $q_{y}$ are the number of half-wavelengths in the $x$ - and $y$-directions of mode $q$.

Substituting eq. (19) into eq. (18), evaluating the partial derivatives, multiplying by the shape function and then integrating over the surface area of the plate and rearranging, yields the following expression for the modal amplitudes

$$
a_{q}\left(\omega_{n}\right)=\frac{4 F^{(n)} \phi_{q}\left(x_{0}, y_{0}\right)}{m L_{x} L_{y}\left[(1+\mathrm{i} \eta) \omega_{q}^{2}-\omega_{n}^{2}\right]}
$$

where 


$$
\omega_{q}=\sqrt{\frac{D}{m}}\left(\left(\frac{q_{x} \pi}{L_{\mathrm{x}}}\right)^{2}+\left(\frac{q_{y} \pi}{L_{\mathrm{y}}}\right)^{2}\right)
$$

is the natural frequency of the $q^{\text {th }}$ mode.

The panel response produces a sound field which is a sum of tones. The sound power from the $n^{\text {th }}$ tone $(n=1,2, \ldots)$ due to excitation by a series of droplet impacts at $x=x_{0}$ and $y=y_{0}$ is given by

$$
\Pi^{(n)}\left(\omega_{n}\right)=2 \int_{0}^{L_{y}} \int_{0}^{L_{x}} \Re\left\{-\mathrm{i} \omega_{n} \widehat{w}^{*}\left(x, y, \omega_{n}\right) \hat{p}\left(x, y, \omega_{n}\right)\right\} \mathrm{d} x \mathrm{~d} y,
$$

where $\hat{p}\left(x, y, \omega_{n}\right)$ is the acoustic pressure on the plate surface at angular frequency $\omega_{n}$ which can be calculated using the Rayleigh integral

$$
\hat{p}\left(x, y, \omega_{n}\right)=\frac{-\omega_{n}^{2} \rho_{0}}{2 \pi} \int_{0}^{L_{y}} \int_{0}^{L_{x}} \widehat{w}\left(x^{\prime}, y^{\prime}, \omega_{n}\right) \frac{\exp \left\{-\mathrm{i} k_{n} R\right\}}{R} \mathrm{~d} x^{\prime} \mathrm{d} y^{\prime},
$$

where $k_{n}=\omega_{n} / c_{0}$ and $R=\sqrt{\left(x-x^{\prime}\right)^{2}+\left(y-y^{\prime}\right)^{2}}$.

Assuming the raindrops from the different holes in the artificial rainfall tray are randomly distributed, an area-average radiated sound power, $\bar{\Pi}^{(n)}\left(\omega_{n}\right)$, is calculated. This is done by averaging $\Pi^{(n)}$ over all possible force locations on the panel surface and summing over all $N$ droplet impact points (recall that the tray has $N$ holes) assuming the raindrops from different holes are uncorrelated i.e.

$$
\bar{\Pi}^{(n)}\left(\omega_{n}\right)=\frac{N}{L_{x} L_{y}} \int_{0}^{L_{y}} \int_{0}^{L_{x}} \Pi^{(n)}\left(\omega_{n}\right) \mathrm{d} x_{0} \mathrm{~d} y_{0} .
$$

Substituting eq. (23) into eq. (25) and utilising eqs. (19) - (21) and (24) yields, after some manipulation,

$$
\bar{\Pi}^{(n)}\left(\omega_{n}\right)=2 N \frac{\rho_{0} c_{0} \omega_{n}^{2}\left|F^{(n)}\right|^{2}}{m^{2} L_{x} L_{y}} \sum_{q=1}^{Q} \frac{\sigma_{q}\left(\omega_{n}\right)}{\left|(1+\mathrm{i} \eta) \omega_{q}^{2}-\omega_{n}^{2}\right|^{2}},
$$

where

$$
\sigma_{q}\left(\omega_{n}\right)=\frac{2 k_{n}}{\pi L_{x} L_{y}} \int_{0}^{L_{y}} \int_{0}^{L_{x}} \int_{0}^{L_{y}} \int_{0}^{L_{x}} \phi_{q}(x, y) \phi_{q}\left(x^{\prime}, y^{\prime}\right) \frac{\sin \left(k_{n} R\right)}{R} \mathrm{~d} x \mathrm{~d} y \mathrm{~d} x^{\prime} \mathrm{d} y^{\prime},
$$

is the radiation efficiency of the $q$ th mode (defined as the real part of eq. (13) in ref. [36] or eq. 2.5 in [40]). This can be evaluated efficiently using the method described in Davy [36]. In the calculations presented in this paper, all modes with natural frequencies less than $10 \mathrm{kHz}$ were included in the modal summation.

\subsection{Radiation model 3}

In this section we present a third method for calculating the radiated sound power. This method accounts for the effect of the number and location of the panel excitation positions. Here we restrict the analysis 
to sound radiation from a thin, homogenous and isotropic flat plate. However, the method could easily be adapted to more complex structures using the modal Transfer Matrix Method (mTMM) presented in ref. [39].

The transverse displacement (in the $z$-direction) of the plate at position $(x, y)$ at angular frequency $\omega_{n}$, is $\widehat{w}\left(x, y, \omega_{n}\right) \exp \left\{\mathrm{i} \omega_{n} t\right\}$. The impact force at angular frequency $\omega_{n}$ due to a train of droplets impacting at one position is $F^{(n)} \exp \left\{\mathrm{i} \omega_{n} t+\mathrm{i} \beta\right\}$ where $F^{(n)}$ is calculated using the method described at the start of this section. These forces are applied at $N$ randomly selected positions with the phase $\beta$ $(0 \leq \beta \leq 2 \pi)$ at each position randomly selected to simulate the fact that the forces are uncorrelated.

The complex amplitudes of the transverse displacements and the forces on the upper surface of the panel at $M(M \geq N)$ different $(x, y)$ locations are collected in the complex vectors $\mathbf{w} \in \mathbb{C}^{\mathrm{M} \times 1}$ and $\mathbf{f} \in \mathbb{C}^{\mathrm{M} \times 1}$. These positions include the locations where the droplet impact forces occur. Thus, $\mathbf{f}$ is a vector of zeros except for the $N$ terms, which correspond to the droplet impact locations. Note that the effect of fluid loading on the plate response is neglected.

It is assumed that $\widehat{w}\left(x, y, \omega_{n}\right)$ is given by eq. (19) with the summation truncated at $q=Q$. The normal displacement vector can therefore be written as

$$
\mathbf{w}=\mathbf{T a},
$$

where $\mathbf{a} \in \mathbb{C}^{\mathrm{Q} \times 1}$ is a vector of modal amplitudes and $\mathbf{T}=\left[\boldsymbol{\phi}_{1}, \boldsymbol{\phi}_{2}, \ldots, \boldsymbol{\phi}_{\mathrm{Q}}\right] \in \mathbb{C}^{\mathrm{M} \times \mathrm{Q}}$ is referred to as the transformation matrix, where $\boldsymbol{\phi}_{q} \in \mathbb{C}^{\mathrm{M} \times 1}$ is a vector of the $q^{\text {th }}$ modal shape function, $\phi_{q}$, evaluated at each of the $M$ different $(x, y)$ positions.

Following the analysis presented in $\S 3.2$, the modal amplitude vector can be calculated as

$$
\mathbf{D}_{d}^{\prime} \mathbf{a}=\mathbf{T}^{\mathrm{T}} \mathbf{f}=\mathbf{f}^{\prime}
$$

where $\mathbf{f}^{\prime}$ is a modal force vector and $\mathbf{D}_{d}^{\prime}=\operatorname{diag}\left[D_{d, 1}, D_{d, 2}, \ldots, D_{d, Q}\right] \in \mathbb{C}^{\mathrm{Q} \times \mathrm{Q}}$ is a modal dynamic stiffness matrix where, for this case,

$$
D_{d, q}=\frac{m L_{x} L_{y}}{4}\left[(1+\mathrm{i} \eta) \omega_{q}^{2}-\omega_{n}^{2}\right]
$$

For more complex panels, an equation of the form of eq. (29) can be derived which relates the modal amplitude vector to the modal force vector using a modal dynamic stiffness matrix. The method for calculating the modal dynamic stiffness matrix using the transfer matrix method is described in ref. [39].

The time-average power radiated from the lower surface of the panel can be calculated using eq. (23). Substituting eq. (24) in eq. (23) and making use of eq. (19) yields

$$
\Pi^{(n)}\left(\omega_{n}\right)=\sum_{q=1}^{Q} \sum_{q^{\prime}=1}^{Q} 2 \Re\left\{-\mathrm{i} \omega_{n} a_{q}^{*} D_{\mathrm{dir}, q^{\prime}, q}^{\prime} a_{q^{\prime}}\right\}=2 \Re\left\{-\mathrm{i} \omega_{n} \mathbf{a}^{\mathrm{H}} \mathbf{D}_{\mathrm{dir}}^{\prime} \mathbf{a}\right\},
$$

where $\mathbf{D}_{\mathrm{dir}}^{\prime}$ is the acoustic dynamic stiffness matrix of the acoustic half-space adjacent to the baffled panel. The individual terms in $\mathbf{D}_{\mathrm{dir}}^{\prime}$ are defined by eq. (32) (which corresponds to eq. (5b) of ref. [41]). 


$$
D_{\mathrm{dir}, q^{\prime}, q}^{\prime}=\frac{-\rho_{0} \omega_{n}^{2}}{2 \pi} \int_{0}^{L_{y}} \int_{0}^{L_{x}} \int_{0}^{L_{y}} \int_{0}^{L_{x}} \phi_{q}(x, y) \frac{\exp \left\{-\mathrm{i} k_{n} R\right\}}{R} \phi_{q^{\prime}}\left(x^{\prime}, y^{\prime}\right) \mathrm{d} x^{\prime} \mathrm{d} y^{\prime} \mathrm{d} x \mathrm{~d} y
$$

Methods for calculating $\mathbf{D}_{\text {dir }}^{\prime}$ are discussed in $\S 2.3$ of [39]. Eq. (31) can also be rewritten as

$$
\Pi^{(n)}\left(\omega_{n}\right)=4 \omega_{n}^{2} \mathbf{a}^{\mathrm{H}} \mathbf{A} \mathbf{a}
$$

where

$$
\mathbf{A}=-\frac{\Re\left\{\mathbf{i}_{\mathrm{dir}}^{\prime}\right\}}{2 \omega_{n}}, \quad A_{q, q^{\prime}}=\frac{\rho_{0} \omega_{n}}{4 \pi} \int_{0}^{L_{y}} \int_{0}^{L_{x}} \int_{0}^{L_{y}} \int_{0}^{L_{x}} \phi_{q}(x, y) \frac{\sin \left(k_{n} R\right)}{R} \phi_{q^{\prime}}\left(x^{\prime}, y^{\prime}\right) \mathrm{d} x^{\prime} \mathrm{d} y^{\prime} \mathrm{d} x \mathrm{~d} y,
$$

is the power transfer matrix defined by Snyder and Tanaka [42]. This is related to the specific radiation resistance matrix, $\mathbf{\Xi}$, defined in eq. (7) of ref. [43] via $\mathbf{A}=\frac{1}{8} \rho_{0} c_{0} L_{x} L_{y} \mathbf{\Xi}$. Note that $\mathbf{A}$ is symmetric and that the diagonal terms $A_{q q}=\frac{1}{8} \rho_{0} c_{0} L_{x} L_{y} \sigma_{q}$, where $\sigma_{q}$ can be calculated efficiently using the formula of Davy [36]. The off-diagonal terms are only non-zero when modes $q$ and $q^{\prime}$ have mode numbers with the same parity in both the $x$ and $y$ directions, which is the case for about a quarter of the total number of terms. In this paper the components of this matrix are calculated using the approximate formula of Snyder and Tanaka (eq. 46 in ref. [42]) for off diagonal terms which comprise contributions from modes which satisfy $\frac{k_{n} L}{\pi q}<0.8$ (where $L=L_{x}$ or $L_{y}$ and $q=q_{x}, q_{y}, q_{x}^{\prime}$ or $q_{y}^{\prime}$ ) in both the $x$ and $y$ directions for both the $q$ and $q^{\prime}$ modes. Other terms are calculated using the exact formula presented in ref. [43]. The power transfer matrix was calculated for all modes with natural frequencies below $10 \mathrm{kHz}$ at the centre frequency of each one-third octave band between $100 \mathrm{~Hz}$ and $5 \mathrm{kHz}$. For the purposes of calculating the sound power using eq. 33, the power transfer matrix calculated for the one-third octave band containing the frequency of interest was used.

As the rain impact is modelled deterministically, the influence of the randomly chosen positions and phases is examined using the Monte Carlo method. This involved calculating the sound power for fifty different realisations with different force locations and phases. These results were used to calculate the mean sound power $\bar{\Pi}^{(n)}$ of the realisations.

\section{Rainfall noise due to heavy rain on a glass pane}

To test the models presented in $\S \S 2-3$, in this section we describe experiments to measure the noise produced by artificial rainfall on a flat panel. For these experiments, we use the method described in the ISO rainfall noise standard [4], [5], for artificial heavy rainfall. Along with the definitions of drop parameters discussed in $\S 2$, the ISO standard specifies the method for generating rainfall, and a reference test specimen which can be used to generate noise levels for comparison with measurements in other laboratories. Here we describe the ISO test method, noting several issues which we encountered implementing the test method. We then use the models presented in $\S \S 2-3$ to calculate the rainfall noise level produced in the standard reference test and compare these to our measured data and a reference sound level included in the standard. We then use the theoretical models to investigate the sensitivity of predicted rainfall noise levels to various parameters.

\subsection{Implementing the ISO rainfall noise standard reference test}

\subsubsection{Artificial rainfall generation}


The artificial heavy rain used in the ISO standard test method is generated by a tank with a perforated base. This tank must be constructed of $1 \mathrm{~cm}$ thick polycarbonate plates with the bottom surface being perforated with $1 \mathrm{~mm}$ diameter holes and reinforced by metal strips. The standard recommends that the base have a minimum area of $1.6 \mathrm{~m}^{2}$ and have approximately 60 holes per $\mathrm{m}^{2}$. The rainfall rate is set by the height of the water in the tank which is kept constant during testing and which requires the tank base to be perfectly horizontal and flat.

We constructed a tank to meet all required specifications, except that our tank base had an area of $0.95 \mathrm{~m}^{2}$ because of the narrow space inside our facility. Our tank did not produce heavy rainfall which met the specifications of the standard. It was observed during testing that the droplets from some holes did not release cleanly, but rather stuck to the underside of the tank close to the hole and produced significantly larger droplets. In order to prevent this occurring, we blocked approximately half of the holes and increased the height of the water in the tank to produce the correct flow rate. The size of the droplets which this configuration produced was assessed by separately measuring the weight of 30 individual droplets falling from 6 different holes and calculating the diameter of an equivalent spherical droplet. The droplet weight was measured using a high accuracy weighing scale (Mettler Type: AE $200-\mathrm{S} \mathrm{s} / \mathrm{n}$. 122281). The average droplet diameter was $6.0 \mathrm{~mm}$ and all droplets had diameters within $0.25 \mathrm{~mm}$ of this value.

Two other papers have reported difficulties generating rainfall matching the nominal characteristics using tanks constructed to the specifications of the ISO standard. Chene, Guigou-Cater and Larsen [31] observed that the detachment of the drop from the tank was "not easy to manage", which they attributed to the quality of the holes and the flatness and levelness of the lower surface of the tank. They recommended the use of individual metal nozzles which they claim produce much more consistent droplets. Yu and Hopkins [21] reported constructing a "rain box" in accordance with the ISO standard. They found that this produced $5.9 \mathrm{~mm}$ diameter droplets. They therefore developed an alternative system which used nozzles and which produced $4.6 \mathrm{~mm}$ diameter droplets. We also note that the standard mentions that a random distribution of holes over the bottom tank surface is preferred to a uniform distribution. However, figure H.1 shows a tank with holes distributed over the bottom surface with each hole apparently randomly located within each rectangle of a rectangular grid pattern.

Given our experience and the reports in refs. [21] and [31], we suggest that further work is required to develop a tank design which consistently produces the artificial rainfall specified in the standard. Until such a design is available we recommend that the size of the droplets produced by a tank operating at the correct rainfall rate be verified prior to testing. This is not currently required by the ISO standard if the tank is constructed to the specifications given in the standard. Although our focus here is on heavy rain, we also constructed a tank to produce intense rain according to the specifications given in the ISO standard. We were also unsuccessful in using this to produce intense rainfall with the correct nominal characteristics.

It is critical that the artificial rainfall tank produces droplets of the correct diameter. This ensures that the droplets have the correct mass, hit the sample with the expected impact velocity and thus have the correct impulse and impact force spectrum. The standard states that for tanks constructed to the specifications given in the standard, the impact velocity can be calculated rather than measured. However, no calculation method is provided. We suggest that eq. (1) using $H$ as the nominal fall height and using measured droplet diameters is appropriate for this purpose. Once the diameter is accurately known then the impact velocity can be accurately calculated using eq. (1). The analytic models predict that, assuming all other variables are held constant, the radiated sound power will scale in proportion to the rainfall rate. We suggest that the rainfall noise standard should allow a range of different flow rates 
to be used during testing, with the levels corrected to a nominal value afterwards. This may allow more control over the droplet generation process to produce droplets with diameters closer to those of the nominal heavy rain droplet.

\subsubsection{The reference test specimen}

The reference test specimen is made up of a single glass pane with a thickness $L_{z}=6 \pm 0.1 \mathrm{~mm}$ and transverse dimensions $L_{x}=1250 \pm 50 \mathrm{~mm} \times L_{y}=1500 \pm 50 \mathrm{~mm}$. The mounting of the glass pane is shown in Annex D of ref. [5] (we note that the 2010 version is referenced in ref. [4]), except for the edge used for water drainage. The pane is mounted between two wooden beads with putty filling the gaps between the glass pane and the surrounding frame. In the tests reported here, the glass pane was mounted as specified in the standard.

Although the standard states that "the use of a thermally toughened safety glass is strongly recommended", the material properties of this glass are not specified. This is a serious shortcoming and makes it difficult to compare results generated using different reference test specimens. We also note that Annex D of ref. [5] describes a glass pane to be used for acoustic testing which is to be a single pane of "soda-lime/silica float glass" with density $\rho_{s}=2500 \mathrm{~kg} \cdot \mathrm{m}^{-3}$ and modulus of elasticity $E=70$ GPa. Assuming a Poisson's ratio $v=0.24$, a $6 \mathrm{~mm}$ thick glass plate with these material properties will have a critical frequency $f_{c}=1983 \mathrm{~Hz}$. However, the glass pane referred to in Annex D has a thickness of $10 \mathrm{~mm}$ so does not correspond to the reference test specimen.

The mounting configuration of the reference test specimen is not clearly stated in the standard. We assume that it is intended that the specimen is to be tested at an inclination of $30^{\circ}$ which is the minimum angle recommended in Annex K of ref. [5] for testing skylights. §H2.2 of ref. [4] also states that the minimum tank area of $1.6 \mathrm{~m}^{2}$ is required in order to totally cover "smaller test specimens in the standard configuration with a $30^{\circ}$ slope". This would include the reference test specimen which measures $1.25 \mathrm{~m} \times 1.5 \mathrm{~m}$. A tank with dimensions of $1.25 \mathrm{~m} \times 1.3 \mathrm{~m}$ (with area $1.625 \mathrm{~m}^{2}$ ) is shown in figure H.1 of ref. [4]. This tank would just provide complete coverage of the reference test specimen mounted at an angle of $30^{\circ}$.

Testing at an angle of inclination of $30^{\circ}$ causes several effects. Firstly, the impact force spectrum will be modified compared to that produced by impact on a horizontal surface as discussed in $\S 2$. Secondly, the inclination angle will affect how easily water drains from the surface. We observed that during testing, the droplets do impact on a slightly wet surface and small puddles can form close to the impact point. This will affect the impact force if a droplet hits these rather than a dry surface. Finally, the impact velocity will vary over the surface of the test specimen. The nominal fall height assumed in the standard is the distance from the bottom of the tank base to the geometric middle of the specimen. In our test at an inclination angle of $30^{\circ}$ we used $H=3.5 \mathrm{~m}$ as recommended in the standard. This resulted in a calculated impact velocity of $7.23 \mathrm{~m} \cdot \mathrm{s}^{-1}$ (using eq. (1)) which is within the $\pm 1 \mathrm{~m} \cdot \mathrm{s}^{-1}$ tolerance allowed by the standard.

\subsubsection{Noise intensity and loss factor measurements}

The standard specifies that rainfall noise levels should be reported in terms of a one-third octave band sound intensity level. This can be determined using one of two different methods: either using direct measurement of the sound intensity or indirectly via measurement of the volume-average sound pressure level in the reverberant space beneath the roof with known volume and reverberation time. The sound intensity levels are related to the theoretical sound power levels via the following equation 


$$
L_{I}=L_{w}-10 \log _{10}\left(\frac{A_{e}}{A_{\text {ref }}}\right)
$$

where $A_{e}$ is the excitation area of the reference test specimen and $A_{\text {ref }}=1 \mathrm{~m}^{2}$. This is taken to be the projection of the tank onto the test specimen. Reference values for $L_{I}$ are given in table I.1 of ref. [4]. The source of these values is not provided and thus it is not clear whether these are levels which are to be expected in testing.

The reference values for $L_{I}$ given in the ISO standard have a peak value in the $2500 \mathrm{~Hz}$ one-third octave band. However, a prediction made of the noise produced by heavy rainfall on a standard reference test specimen with the material properties given above will have a peak value in the $2000 \mathrm{~Hz}$ one-third octave band. In $\S 4.2$ we show that an otherwise identical pane to that described above, but with $E=50 \mathrm{GPa}$ (for which $f_{c}=2347 \mathrm{~Hz}$ ), will produce an $L_{I}$ spectrum with a peak value in the $2500 \mathrm{~Hz}$ one-third octave band. This value of $E$ was selected to produce a good match between the frequency of the predicted and reference peak $L_{I}$ values. These results suggest that the glass pane used to generate the data in table I.1 of ref. [4] may have had different material properties from those listed above. As we will show later, our experimental results show a peak $L_{I}$ value in the $2000 \mathrm{~Hz}$ one-third octave band. Hopkins (fig. 4.75 in ref. [11]) also presents one-third octave band sound power levels produced by rainfall onto a $6 \mathrm{~mm}$ glass pane in which the peak level occurs in the $2000 \mathrm{~Hz}$ one-third octave band. (Hopkins also states that $f_{c}=2021 \mathrm{~Hz}$ for the pane used in that test).

The ISO standard states that in order "to calibrate the mounting conditions of the reference test specimen, the structural decay time should be measured according to ISO 10140-4, from which the total loss factor, $\eta$ (in the one-third octave band with centre frequency, $f_{c}$ ) is calculated from the structural decay time, $T_{s}$, using $\eta=2.2 /\left(f_{c} T_{s}\right)$ ". Reference values for $\eta$ are given in table I.1 of ref. [4]. The source of these values is not provided. ISO 10140-4 states that the total loss factor should be measured according to ISO/DIS 10848-1, 7.3. The small size of the standard reference specimen means that the guidance on the location and number of measurement and excitation positions stated in that standard cannot be fulfilled. In the measurements reported here we used 2 measurement positions and two excitation positions for each measurement position with excitation using an impact hammer. The total loss factor calculated using this approach is shown in figure 13 which also shows the reference values from the standard. 


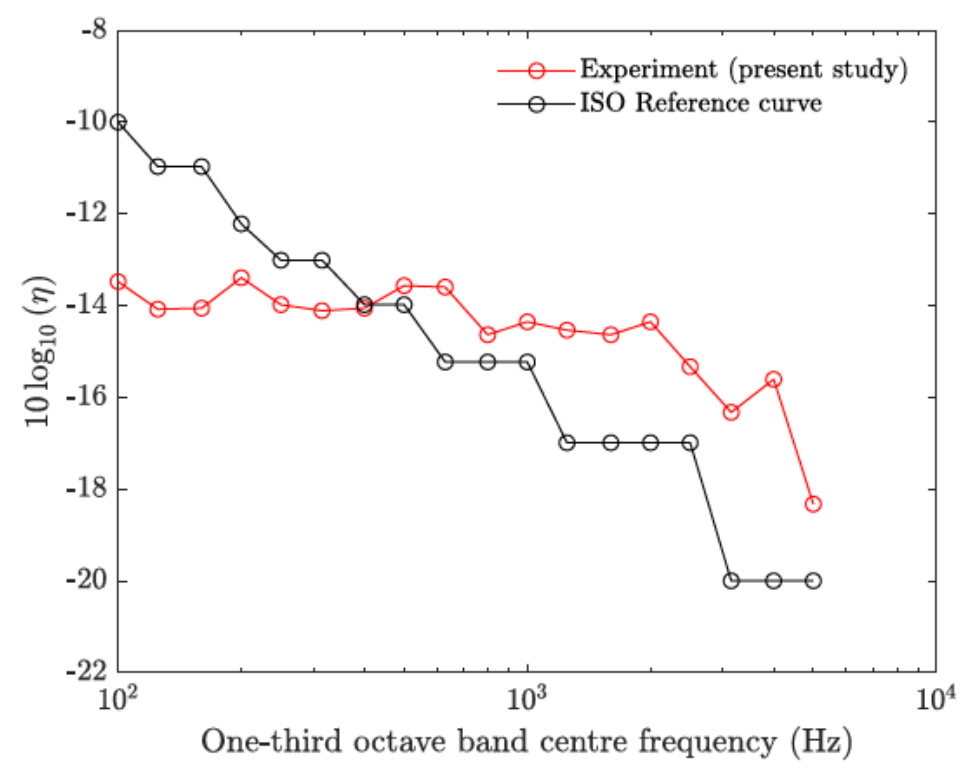

Figure 13. Total loss factor in one-third octave bands. Measured values compared with the ISO reference values (taken from table I.1 in ref. [4]).

\subsubsection{Experimental results}

The sound intensity levels produced during our test are shown in figure 14 (right). These may be compared with the ISO reference curve values which are shown in figure 14 (left). Predictions are also made for both cases using the theoretical models presented in this paper. The heavy rainfall impact force model and radiation model 1 are used for these predictions. The ISO reference curve predictions are made assuming $5 \mathrm{~mm}$ diameter droplets impacting at $7 \mathrm{~m} \cdot \mathrm{s}^{-1}$ onto the standard reference sample with an assumed Young's modulus of $50 \mathrm{GPa}$ and a total damping factor equal to that of the ISO reference sample loss factor. For predictions of our experiment we used $6 \mathrm{~mm}$ diameter droplets impacting at $7.23 \mathrm{~m} \cdot \mathrm{s}^{-1}$ onto our standard reference sample with the measured total damping loss factor. $A_{e}$ was set equal to the area of our tank projected onto the standard reference sample. For the ISO reference case, the predictions are in modest agreement with the reference curve being $6 \mathrm{~dB}$ lower than the reference curve at $100 \mathrm{~Hz}$ and over-predicting by $3 \mathrm{~dB}$ at the critical frequency. There is very good agreement between the predictions and the measurements made using our rig below the critical frequency, however, the intensity levels at and above the critical frequency are not well predicted.

Our experimentally measured levels are somewhat different to those of the ISO reference curve. According to the theoretical model, this is due to the different loss factors and Young's moduli of the two reference specimens and the different rainfall used in both tests. The ISO standard also defines a corrected one-third octave band intensity level defined by

$$
L_{I, m}=L_{I}+10 \log _{10}\left(\frac{\eta}{\eta_{\mathrm{ref}}}\right) .
$$

This corrected level appears to be intended to try to correct for the different total loss factors which different rigs will inevitably produce. Such a correction would be based on the assumption that the measured intensity level is inversely proportional to $\eta$. According to eq. (16), we expect this to be the case at frequencies above the critical frequency, but not necessarily below the critical frequency where the non-resonant field contribution can be significant. 

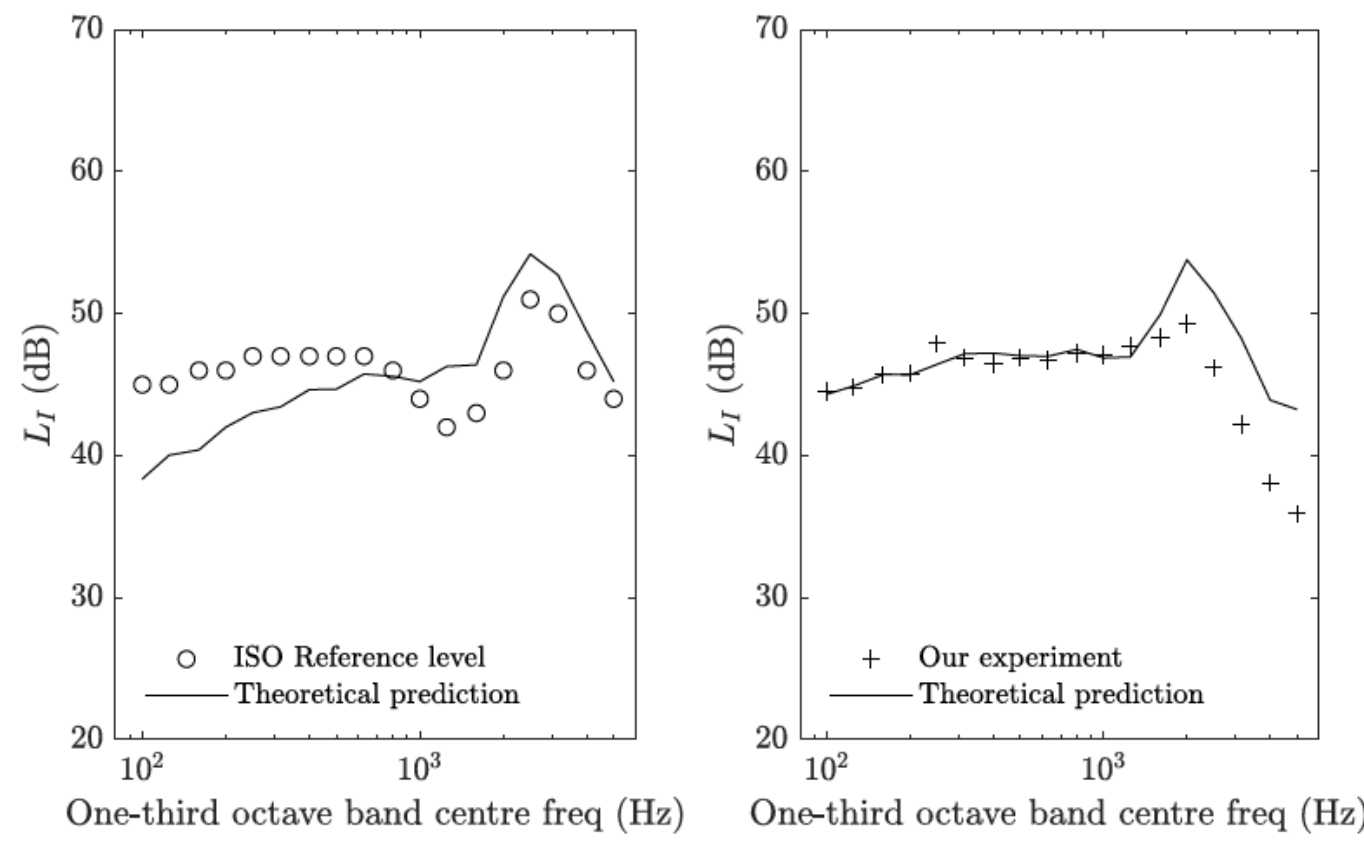

Figure 14. Rain noise sound intensity level, $L_{I}\left(\mathrm{~dB}\right.$ ref. $\left.10^{-12} \mathrm{~W} \cdot \mathrm{m}^{-2}\right)$, for heavy rain on the standard reference sample inclined at $30^{\circ}$. The ISO reference curve (circles), our measurements (crosses) and predictions (lines)

We also conducted an experiment measuring the noise produced by heavy rainfall on the standard reference test specimen inclined at $5^{\circ}$. This specimen was mounted differently to the specimen tested at $30^{\circ}$ and therefore had different total loss factor values, which were measured using the same procedure described above. Because of the shallower angle of inclination for this case and the inability to easily move the rain tank in our laboratory, the fall height for this case was $H=3.81 \mathrm{~m}$ and the impact velocity was calculated as $U_{0}=7.46 \mathrm{~m} \cdot \mathrm{s}^{-1}$. There is excellent agreement between the measurements and predictions which are shown in figure 15. The effect of angle of incidence of the sample on the rain noise level will be explored in more detail in §4.2. 


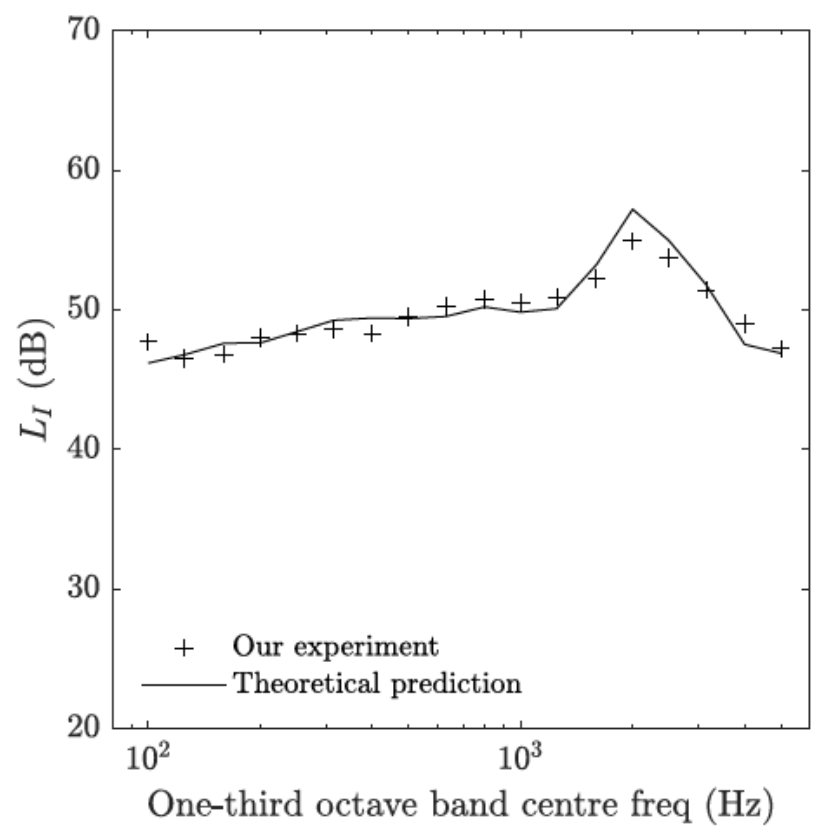

Figure 15. Predicted and measured rain noise sound intensity level, $L_{I}\left(\mathrm{~dB}\right.$ ref. $10^{-12} \mathrm{~W} \cdot \mathrm{m}^{-2}$ ) for heavy rain on the standard reference specimen inclined at $5^{\circ}$.

\subsection{A study of rainfall noise using the theoretical models}

In this section, the theoretical models presented in $\S \S 2-3$ are used to predict the rainfall noise levels produced by heavy rain on the ISO standard reference test specimen inclined at $30^{\circ}$. Unless otherwise stated, we consider this sample with identical material properties to those assumed in the previous section ( $E=70 \mathrm{GPa}, v=0.24$ and total loss factor values given in ref. [4]) and make use of the heavy rainfall noise impact force model and radiation model 1.

Figure 16 plots the $L_{I}$ spectrum predicted using the three different radiation models. The levels predicted by all three models are in good agreement with each other. Radiation model 1 evaluates almost instantaneously in comparison to models 2 and 3 which require significantly more computational power for little gain in accuracy. However, models 2 and 3 could be useful for analysing other structures for which finite size effects are important or which have complicated structures which require a more sophisticated modelling approach. 


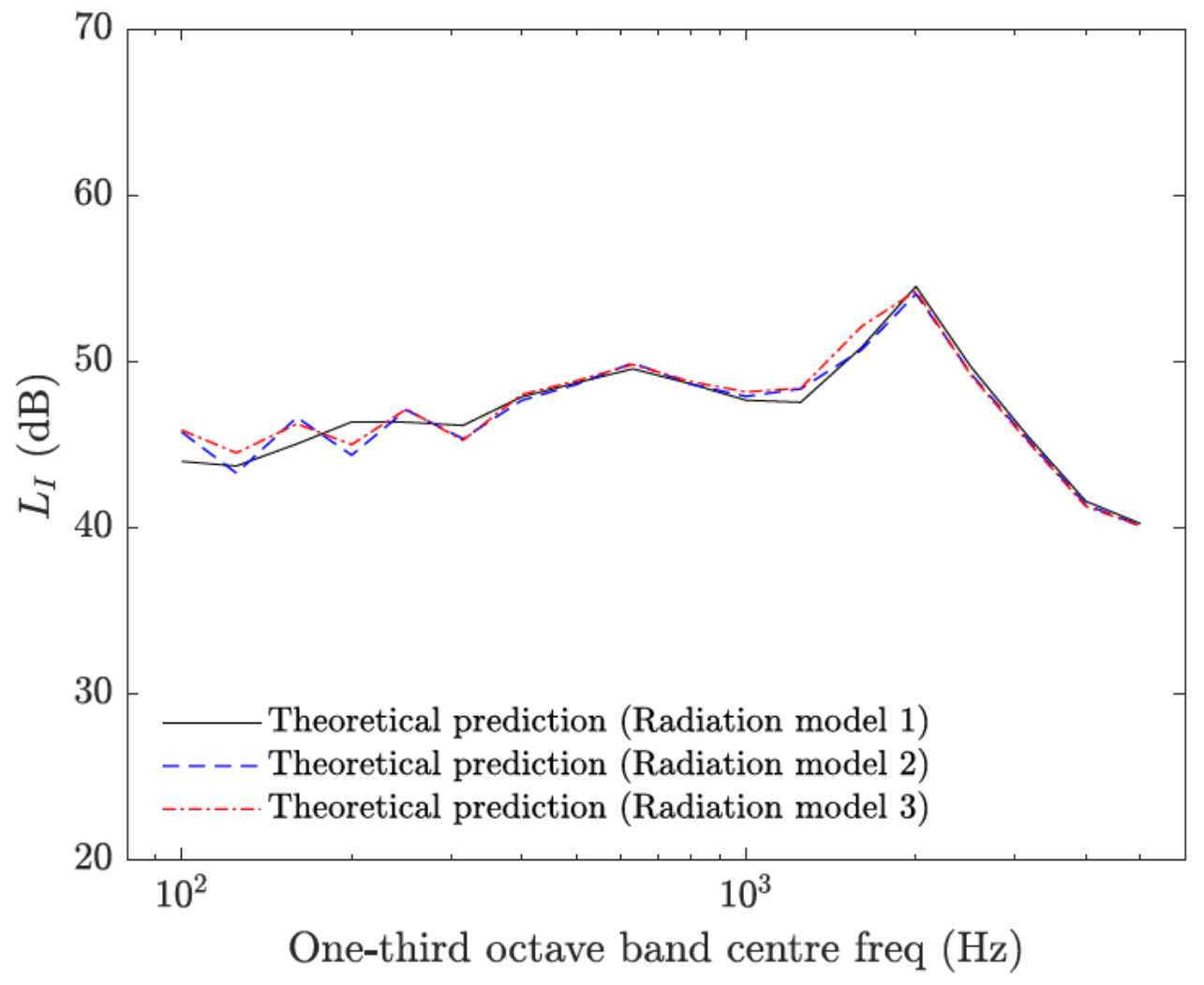

Figure 16. Predicted rain noise sound intensity level, $L_{I}\left(\mathrm{~dB}\right.$ ref. $\left.10^{-12} \mathrm{~W} \cdot \mathrm{m}^{-2}\right)$ for rainfall on the standard reference specimen calculated using three different radiation models: radiation model 1 (black solid line), radiation model 2 (blue dashed line) and radiation model 3 (red dash-dot line).

Figure 17 plots the $L_{I}$ spectrum predicted using the different impact force models. These models include the heavy rainfall impact force model, Yu and Hopkins' empirical model (scaled to account for the slightly different droplet size) and the paraboloidal droplet model commonly used in previous rain noise prediction methods. Note that no scaling is applied to the paraboloidal droplet model to account for incidence effects. There is generally good agreement between the heavy rainfall impact force model and Yu and Hopkins' scaled model with the predictions being within $2 \mathrm{~dB}$ over the entire spectrum (and often within much closer agreement). Surprisingly, the prediction made using the paraboloidal model is also in reasonable agreement with the other two models. This agreement is somewhat coincidental, as the paraboloidal model does not take the effect of angle of incidence into account. A prediction at e.g. $5^{\circ}$ incidence will produce a much larger discrepancy between the models. 


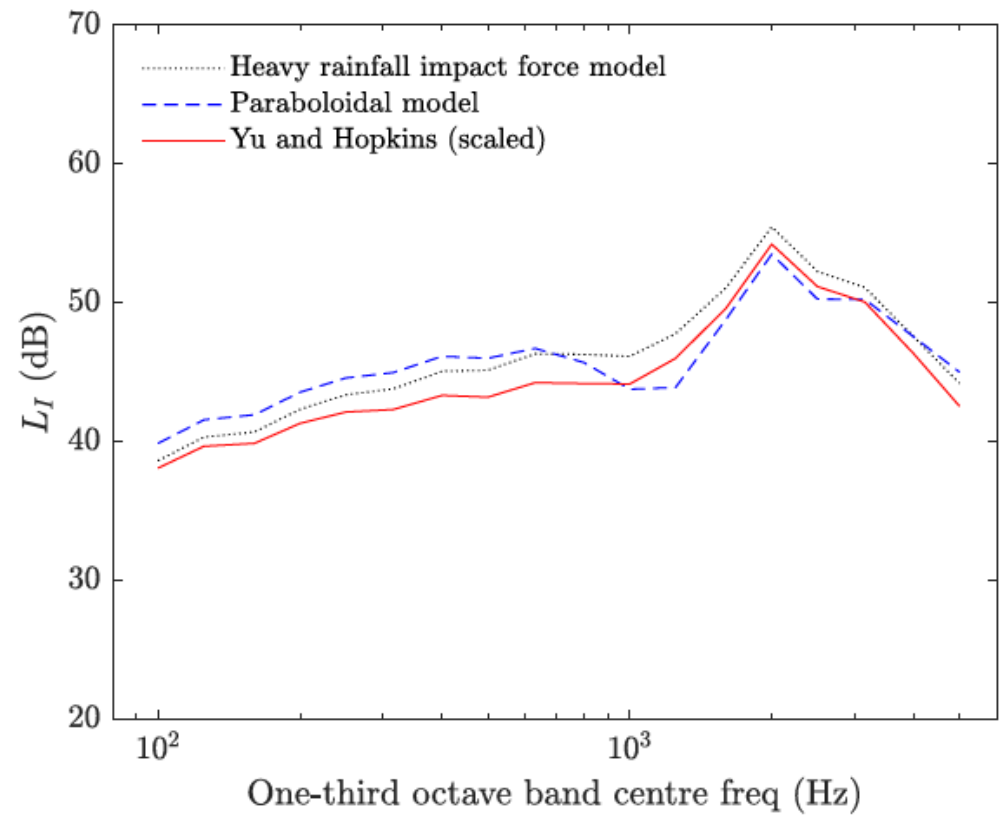

Figure 17. Predicted rain noise sound intensity level, $L_{I}\left(\mathrm{~dB}\right.$ ref. $\left.10^{-12} \mathrm{~W} \cdot \mathrm{m}^{-2}\right)$ for rainfall on the standard reference specimen calculated using different impact force models: heavy rainfall impact force model (black dotted line), Paraboloidal model (blue dashed line) and $\mathrm{Yu}$ and Hopkins empirical model (red solid line).

Figure 18 plots the $L_{I}$ spectrum predicted for different incidence angles. We have ignored the effect of the variable impact velocity over the surface of the sample. Recall that these predictions are made using the heavy rainfall impact force model, which was shown to produce single droplet impact force spectra which were in reasonable agreement with measured data for angles of incidence of $5^{\circ}$ and $30^{\circ}$ (see Figure 12). It has also been assumed that the height of the tank above the sample is adjusted to ensure that the impact velocity is equal to $7 \mathrm{~m} \cdot \mathrm{s}^{-1}$ at the mid-height of the specimen as required by the ISO standard. There is a $2-3 \mathrm{~dB}$ reduction in level produced by increasing the angle of inclination from $0^{\circ}$ to $30^{\circ}$ and a significant further $2-5 \mathrm{~dB}$ reduction by increasing the angle of inclination from $30^{\circ}$ to $45^{\circ}$. This suggests that the angle of inclination is important in determining the rainfall noise level. We suggest that the ISO standard should provide stricter guidance regarding the angles at which specimens may be tested. Currently, the ISO standard requires that the surface being tested is inclined at a minimum angle of " 5 " for roofs and $30^{\circ}$ for skylights. The slope used shall be the lowest that is feasible to ensure water drainage". This discretion could result in roofs being tested at different angles in different testing laboratories. 


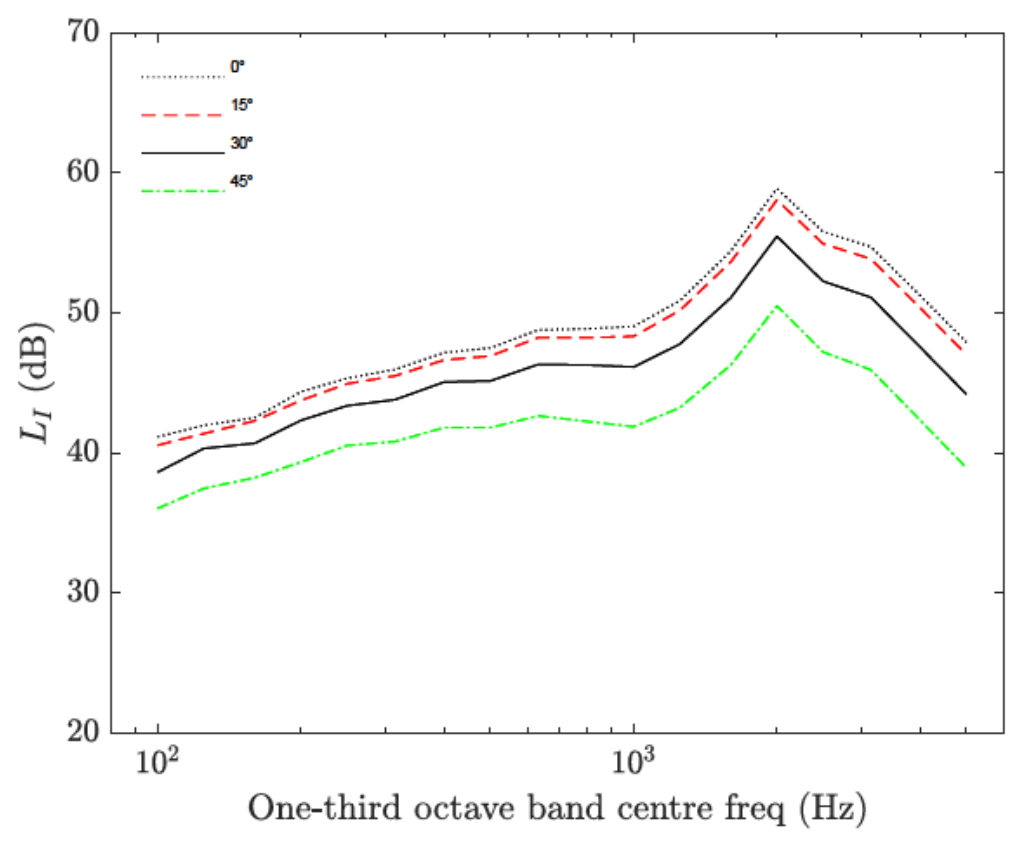

Figure 18. Predicted rain noise sound intensity level, $L_{I}\left(\mathrm{~dB}\right.$ ref. $\left.10^{-12} \mathrm{~W} . \mathrm{m}^{-2}\right)$ for rainfall on the standard reference specimen inclined at different angles $\left(\theta=0^{\circ}, 15^{\circ}, 30^{\circ}\right.$ and $\left.45^{\circ}\right)$.

Above it was noted that the ISO standard does not require the artificial raindrop size or impact velocity to be measured for tanks which are constructed to the specifications given in the standard. In order to assess the likely errors which could occur if the tank produced raindrops of an incorrect size, and where the tank height was calculated assuming the nominal raindrops were produced (i.e. $H=3.5 \mathrm{~m}$ ), the $L_{I}$ spectrum was predicted for a range of different droplet sizes using a drop height of $3.5 \mathrm{~m}$. Note that the heavy rainfall impact force model was used for these predictions. This model does not account for the change in droplet shape which will occur for larger droplets. However the model includes the effect of droplet size using the scaling formula of eq. (2) and the effect of a change in impact velocity calculated using eq. (1). The results of these calculations are shown in figure 19 and show that small changes in droplet diameter of just $1 \mathrm{~mm}$ from the nominal value can produce significant changes (up to $3 \mathrm{~dB}$ ) in the calculated noise levels. This supports our suggestion that the droplet sizes produced by a tank should be carefully checked prior to testing using the ISO standard method. 


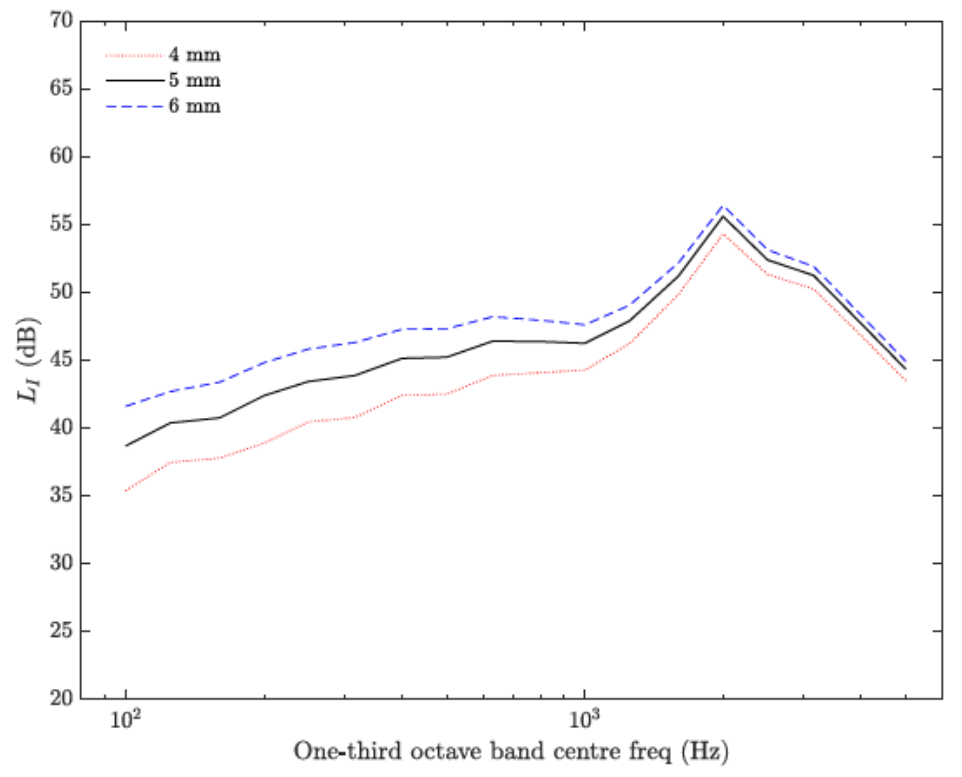

Figure 19. Predicted sound intensity level, $L_{I}\left(\mathrm{~dB}\right.$ ref. $\left.10^{-12} \mathrm{~W} \cdot \mathrm{m}^{-2}\right)$ for rainfall on the standard reference specimen calculated for different droplet sizes.

The ISO test method also allows for tests to be conducted using intense rain. Because of the relatively small drop height $(1 \mathrm{~m})$ required to produce the correct impact velocity $\left(4 \mathrm{~m} \cdot \mathrm{s}^{-1}\right)$, tests using intense rainfall are best undertaken on samples with small angles of incidence (where there is only a small variation in drop height over the sample). Figure 20 plots the sound intensity level produced by heavy and intense rainfall onto a reference test specimen inclined at $5^{\circ}$. The levels produced by intense rain are very low, particularly at low frequencies. This may make testing difficult in laboratories with a high background noise level.

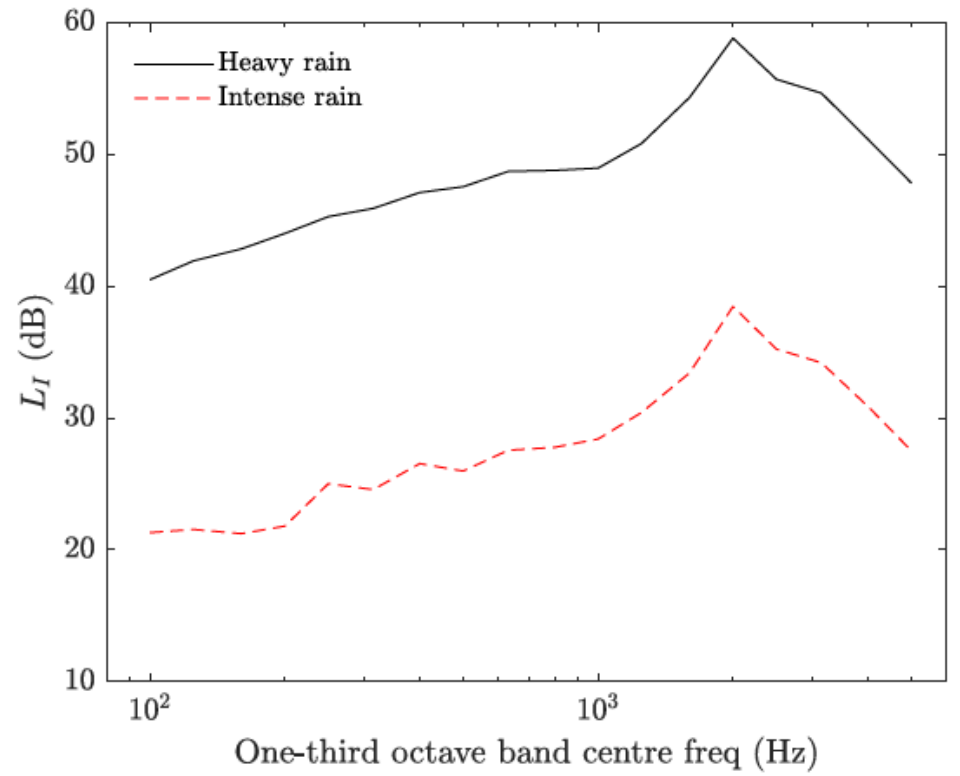

Figure 20. Predicted sound intensity level, $L_{I}\left(\mathrm{~dB}\right.$ ref. $\left.10^{-12} \mathrm{~W} \cdot \mathrm{m}^{-2}\right)$ for heavy and intense rainfall on the standard reference test specimen inclined at $5^{\circ}$. 


\section{Conclusions and recommendations}

Several different models for the force produced by a water droplet impacting on a flat inclined surface have been presented. These models are valid for the nominal intense and heavy raindrops described in the ISO rainfall noise test standard and have been validated against experimental measurements. The best-performed models have been incorporated into theoretical methods for predicting the sound power produced by rainfall on a flat inclined panel. Three methods for predicting the radiated sound power have been presented. The first is valid for a homogeneous panel and allows rapid noise predictions to be made. The second is valid for an isotropic, homogeneous panel and includes the effect of the modal response of the panel. The third method also includes the effect if the modal response of the panel and could be used to model the sound radiation from panels with more complicated structures. All three models were shown to produce very similar predictions for the noise produced by heavy rainfall on the standard reference specimen described in the ISO standard.

An experimental study has investigated the noise produced by heavy rainfall on the standard reference test specimen using the test procedure, described in the ISO standard. The experimentally measured levels were compared with theoretical predictions and showed moderate agreement. The theoretical models were then used to investigate the effect of different parameters on rainfall noise for the purpose of illustrating potential sources of error during testing using the ISO standard method.

We described some of the issues we faced implementing the ISO standard test method. With the aim of increasing consistency, we conclude by summarising our suggestions for future work to investigate and improve this method:

- Droplet size should be clearly defined. The nominal heavy raindrop is not spherical and therefore referring to a diameter is confusing. We suggest that the diameter be interpreted as being equal to that of a spherical droplet of the same volume, consistent with refs. [31] and [21].

- The rainfall type names should be consistent with IEC 60721-2-2, or reference to that standard should be removed.

- It may be worth considering removing the intense rainfall test if it is not mandatory.

- We and other investigators have had great difficulty constructing a tank to the specifications given in the ISO standard which produced droplets with the correct characteristics. Until a robust tank design is available, we suggest that the droplet sizes produced by any tank be carefully measured prior to testing.

- It may be helpful to allow testing at different rainfall rates in order to ensure that the tank produces droplets of the correct diameter. Rainfall noise levels can be easily adjusted to account for different rainfall rates, but noise levels have a much more complicated scaling with droplet size.

- The standard suggests that the droplet impact velocity can be calculated, but does not specify a method for doing so. We suggest that the standard should recommend a method and that eq. (1) is suitable for this purpose.

- The ISO standard describes a reference test specimen which is suitable for generating results for comparison with those generated in different laboratories. Using this specimen in a Round Robin experiment would be useful for assessing the repeatability of the test method.

- The mounting condition for the reference test specimen needs to be clearly specified. We assume that the specimen should be tested at an angle of inclination of $30^{\circ}$. 
- The material properties of the reference test specimen should be clearly specified. These should ideally be consistent with the properties of glass specified in Annex D of ref. [5].

- The source of the reference sound intensity levels and total loss factors in the ISO standard should be stated.

- The method for measuring the total loss factors for small specimens should be clarified.

- We noticed during testing that small puddles could form close to droplet impact locations which could affect the impact force if droplets impact on these rather than on the dry surface. The significance of these puddles depends on the inclination angle of the surface. However, the droplet impact force is also strongly dependent on the inclination angle of the surface. Some judgement is therefore required as to what angle of incidence is most appropriate to allow sufficient drainage. However allowing tests at different angles makes comparisons of different tests difficult. It would be better if the ISO standard specified exact inclination angles at which different surfaces should be tested in order to allow comparison between results from different laboratories.

\section{REFERENCES}

[1] K. O. Ballagh, "Noise of simulated rainfall on roofs," Appl. Acoust., vol. 31, no. 4, pp. 245264, Jan. 1990.

[2] New Zealand Ministry of Education, Designing quality learning spaces: Acoustics, version 2.0. 2016.

[3] UK Department of Education and Education Funding Agency, Building Bulletin 93: acoustic design of schools - performance standards (v. 17). UK, 2015.

[4] ISO 10140-5:2010/Amd. 1:2014. Acoustics - Laboratory measurement of sound insulation of building elements - part 5: Requirements for test facilities and equipment. Amendment 1: rainfall sound. 2014.

[5] ISO 10140-1:2016 Acoustics - Laboratory measurement of sound insulation of building elements - Part 1: Application rules for specific products. 2016.

[6] P. Dubout, "The sound of rain on a steel roof," J. Sound Vib., vol. 10, no. 1, pp. 144-150, Jul. 1969.

[7] J. McLoughlin, D. J. Saunders, and R. D. Ford, "Noise generated by simulated rainfall on profiled steel roof structures," Appl. Acoust., vol. 42, no. 3, pp. 239-255, Jan. 1994.

[8] H. Suga and H. Tachibana, "Sound Radiation Characteristics of Lightweight Roof Constructions Excited by Rain," Build. Acoust., vol. 1, no. 4, pp. 249-270, 1994.

[9] G. Vermeir, P. Mees, and I. Bosmans, "Prediction of rainfall noise on single and double glazing," in proceedings of Internoise 96, 1996.

[10] C. Guigou-Carter, C. , Villot, M., Horlaville, "Study of simulated rainfall noise on roofs and glazings," in proceedings of Forum Acusticum 2002, 2002.

[11] C. Hopkins, Sound Insulation. Butterworth-Heinemann, 2007.

[12] D. Griffin and K. Ballagh, "A consolidated theory for predicting rain noise," Build. Acoust., vol. 19, no. 4, pp. 221-248, 2012.

[13] M. Toyoda and D. Takahashi, "Reduction of rain noise from Ethylene/TetraFluoroEthylene membrane structures," Appl. Acoust., vol. 74, no. 12, pp. 1309-1314, Dec. 2013. 
[14] X. Yan, S. Lu, and J. Li, "Experimental studies on the rain noise of lightweight roofs: Natural rains vs artificial rains," Appl. Acoust., vol. 106, pp. 63-76, May 2016.

[15] B. Petersson, "On the structural acoustic effects of liquid drop impacts." Lund Institute of Technology Report TVBA-3020, 1985.

[16] B. A. T. Petersson, "The liquid drop impact as a source of sound and vibration," Build. Acoust., vol. 2, no. 4, pp. 585-621, 1995.

[17] L. Gordillo, T.-P. Sun, and X. Cheng, "Dynamics of drop impact on solid surfaces: evolution of impact force and self-similar spreading," J. Fluid Mech., vol. 840, pp. 190-214, Apr. 2018.

[18] B. Zhang, Q. Lv, P. Guo, and J. Li, "Experimental study of impact force of a low-speed droplet colliding on the solid surface at different impact angles," in ASME International Mechanical Engineering Congress and Exposition, Proceedings (IMECE), 2017, vol. 7, p. V007T09A022.

[19] Y. Yu and C. Hopkins, "Experimental determination of forces applied by liquid water drops at high drop velocities impacting a glass plate with and without a shallow water layer using wavelet deconvolution," Exp. Fluids, vol. 59, no. 5, p. 84, Apr. 2018.

[20] B. R. Mitchell, J. C. Klewicki, Y. P. Korkolis, and B. L. Kinsey, "The transient force profile of low-speed droplet impact: measurements and model," J. Fluid Mech., vol. 867, pp. 300-322, May 2019.

[21] Y. Yu and C. Hopkins, "Empirical models for the structure-borne sound power input from artificial and natural rainfall," Appl. Acoust., vol. 162, no. 107199, 2020.

[22] M. Nearing and J. M. Bradford, "Relationships Between Waterdrop Properties and Forces of Impact," Soil Sci. Soc. Am. J., vol. 51, no. 2, pp. 425-430, 1987.

[23] M. Nearing, J. M. Bradford, and R. Holtz, "Measurement of Force vs. Time Relations for Waterdrop Impact," Soil Sci. Soc. Am. J., vol. 50, no. 6, pp. 1532-1536, 1986.

[24] A. Sahaya Grinspan and R. Gnanamoorthy, "Impact force of low velocity liquid droplets measured using piezoelectric PVDF film," Colloids Surfaces A Physicochem. Eng. Asp., vol. 356, no. 1-3, pp. 162-168, Mar. 2010.

[25] J. Li, B. Zhang, P. Guo, and Q. Lv, "Impact force of a low speed water droplet colliding on a solid surface," J. Appl. Phys., vol. 116, no. 21, p. 214903, Dec. 2014.

[26] D. Soto, A. B. De Larivière, X. Boutillon, C. Clanet, and D. Quéré, "The force of impacting rain," Soft Matter, vol. 10, no. 27, pp. 4929-4934, 2014.

[27] B. Zhang, J. Li, P. Guo, and Q. Lv, "Experimental studies on the effect of Reynolds and Weber numbers on the impact forces of low-speed droplets colliding with a solid surface," Exp. Fluids, vol. 58, no. 9, 2017.

[28] R. J. M. Craik, Sound transmission through buildings using Statistical Energy Analysis. Gower, 1996.

[29] M. Villot, C. Guigou-Carter, and L. Gagliardini, "Predicting the acoustical radiation of finite size multi-layered structures by applying spatial windowing on infinite structures," J. Sound Vib., vol. 245, no. 3, pp. 433-455, Aug. 2001.

[30] L. Gagliardini, "Simulation numérique de la transmission acoustique par les parois simples et multiples," PhD thesis, INSA, Lyon., 1991. 
[31] J. B. Chéné, C. Guigou-Carter, and M. L. Larsen, "Rainfall noise measurements: Assessment and perspective four years after the publication of en ISO 140-18," in Proceedings of 39th International Congress on Noise Control Engineering 2010, INTER-NOISE 2010, 2010, vol. 1, pp. 439-450.

[32] F. M. White, Fluid Mechanics, 4th ed. McGraw-Hill, 1999.

[33] K. Range and F. Feuillebois, "Influence of Surface Roughness on Liquid Drop Impact," $J$. Colloid Interface Sci., vol. 203, no. 1, pp. 16-30, Jul. 1998.

[34] M. E. Clift, R.; Grace, J. R.; Weber, Bubbles, drops and particles. Academic Press, 1978.

[35] J. Philippi, P.-Y. Lagrée, and A. Antkowiak, "Drop impact on a solid surface: short-time selfsimilarity,” J. Fluid Mech., vol. 795, pp. 96-135, May 2016.

[36] J. L. Davy, D. J. Larner, R. R. Wareing, and J. R. Pearse, "The acoustic radiation impedance of a rectangular panel," Build. Environ., vol. 92, pp. 743-755, Oct. 2015.

[37] J. L. Davy, "Sound transmission of cavity walls due to structure borne transmission via point and line connections," J. Acoust. Soc. Am., vol. 132, no. 2, pp. 814-821, Aug. 2012.

[38] J. L. Davy, J. P. Mahn, C. Guigou-Carter, and M. Villot, "The prediction of flanking sound transmission below the critical frequency," J. Acoust. Soc. Am., vol. 132, no. 4, pp. 2359 2370, Oct. 2012.

[39] C. Decraene, A. Dijckmans, and E. P. B. Reynders, "Fast mean and variance computation of the diffuse sound transmission through finite-sized thick and layered wall and floor systems," J. Sound Vib., vol. 422, pp. 131-145, 2018.

[40] F. G. Leppington, E. G. Broadbent, and K. H. Heron, "Acoustic radiation efficiency of rectangular panels," in Proceedings of The Royal Society of London, Series A: Mathematical and Physical Sciences, 1982, vol. 382, no. 1783, pp. 245-271.

[41] R. S. Langley, "Numerical evaluation of the acoustic radiation from planar structures with general baffle conditions using wavelets," J. Acoust. Soc. Am., vol. 121, no. 2, pp. 766-777, 2007.

[42] S. D. Snyder and N. Tanaka, "Calculating total acoustic power output using modal radiation efficiencies," J. Acoust. Soc. Am., vol. 97, no. 3, pp. 1702-1709, 1995.

[43] W. L. Li, "An analytical solution for the self- and mutual radiation resistances of a rectangular plate," J. Sound Vib., vol. 245, no. 1, pp. 1-16, 2001. 


\section{Appendix A. Pressure sensor calibration}

The pressure sensor used in the low-speed droplet impact testing described in $\$ 2.3 .1$ (PCB 106B51) was calibrated using an impact hammer with a vinyl tip (PCB 086E90). Between 8-10 repeated impacts were performed by hand, both on the sensor without any tape and with various layers of tape applied to the top of the sensor. Both signals were sampled at $125 \mathrm{kHz}$ (a lower sampling rate was used as this was the highest achievable with two channels connected to the data acquisition system). The data was smoothed using a rolling average of 10 data points to remove fluctuations (particularly from the less sensitive impact hammer signal). The signal from the calibrated impact hammer was compared to the signal from the pressure sensor to determine a calibration factor. These calibration factors were calculated either using the peak value of the signal from both transducers during the impact or using the integrated area beneath the transducer signal time-history during the impact. Both methods yielded almost identical calibration constants. Figure A1 plots the impact force measured using the calibrated pressure sensor and compares it with that measured by the impact hammer. There is excellent agreement between both curves.

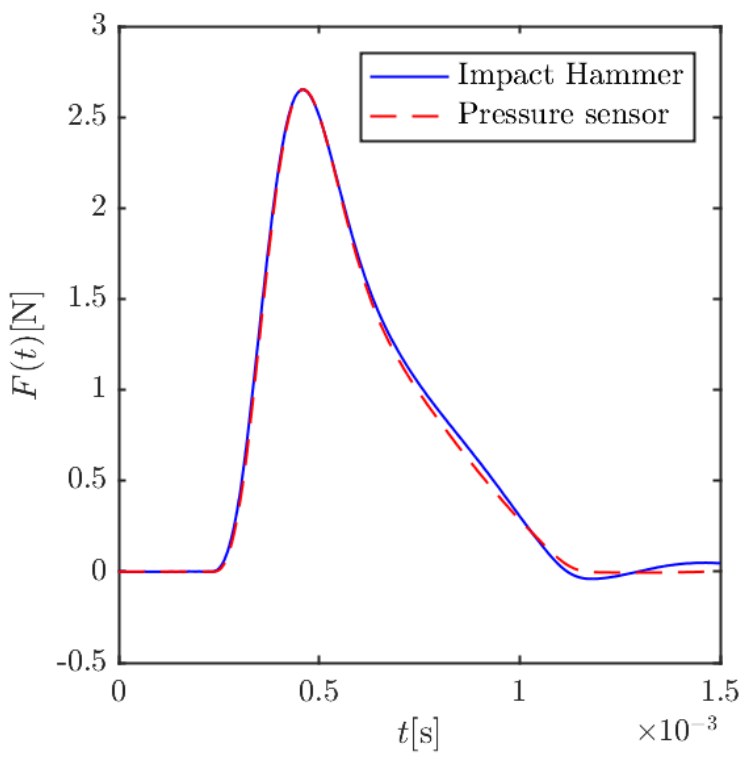

Figure A1. Example agreement between shape of pressure sensor signal (converted to force by comparing peak of signal to impact hammer signal peak) and impact hammer (converted to force with known calibration), for the case of 1 tape layer.

As further verification of the calibration method, droplet momentum was compared to droplet impulse for spherical droplets (with aspect ratio $e>0.93$ ), as shown in figure A2. As discussed in $\$ 2.3 .1$, aiming droplets for $H>50 \mathrm{~cm}$ was difficult due to the small sensor diameter and the fact that droplets at high velocities rarely land with repeatable positioning because of their own unsteady wakes [26]. To overcome these issue multiple trials were conducted and camera footage was used to confirm drop aim. Hence, only the droplets impacting on the approximate centre of the sensor are included here. 


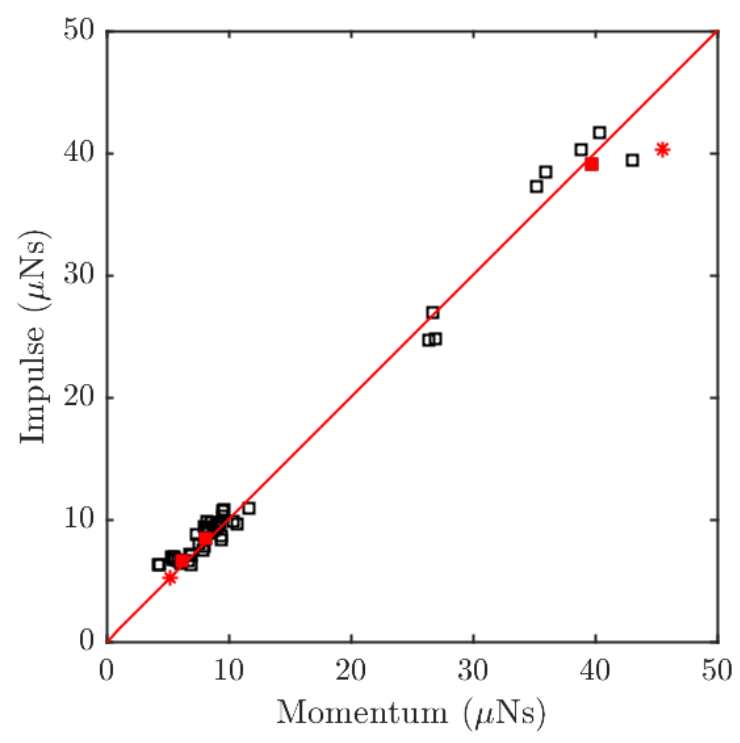

Figure A2. The relationship between change in momentum $\left(m U_{0}\right.$, where $m$ is the droplet mass) and drop impulse estimated from the area under force-time curves. Red squares are the droplets included in figures 6-7 shown in the body of the paper. Red stars are angled ( $30^{\circ}$ incline) measurements included in the body of the paper in figures 9-10. In the case of angled incline the normal component of velocity is used in the momentum calculation. Black hollow squares are other trials with silicone oil and water given to show the general trend (only spherical droplets $0.93<\mathrm{e}<1.0$ are shown). The droplet mass is estimated by $m=\frac{\pi}{6 \rho D^{3}}$, where drop diameter is estimated from the drop length projected onto the axis symmetry $\left(D_{v}\right)$ and drop length perpendicular to this axis $\left(D_{h}\right)$, such that $D=\left(D_{h} D_{v}^{2}\right)^{1 / 3}$. 